Accepted to ApJ

Preprint typeset using $\mathrm{LAT}_{\mathrm{EX}}$ style emulateapj v. 04/03/99

\title{
THE DISK AND DARK HALO MASS OF THE BARRED GALAXY NGC 4123.
}

\section{OBSERVATIONS}

\author{
BENJAMIN J. WEINER ${ }^{1,2}$ \\ Observatories of the Carnegie Institution of Washington, 813 Santa Barbara St, Pasadena, CA 91101 \\ T. B. Williams ${ }^{1}$ \\ Department of Physics and Astronomy, Rutgers University, 136 Frelinghuysen Rd., Piscataway, NJ 08854 \\ J. H. VAN GORKOM ${ }^{2}$ \\ Department of Astronomy, Columbia University, 538 W. 120th St., New York, NY 10227 \\ AND \\ J. A. SEllwood \\ Department of Physics and Astronomy, Rutgers University, 136 Frelinghuysen Rd., Piscataway, NJ 08854 \\ Accepted to ApJ
}

\begin{abstract}
The non-circular streaming motions in barred galaxies are sensitive to the mass of the bar and can be used to lift the degeneracy between disk and dark matter halo encountered when fitting axisymmetric rotation curves of disk galaxies. In this paper, we present photometric and kinematic observations of NGC 4123 , a barred galaxy of modest size $\left(V_{\text {rot }}=130 \mathrm{~km} \mathrm{~s}^{-1}, L=0.7 L_{*}\right)$, which reveal strong noncircular motions. The bar has straight dust lanes and an inner Lindblad resonance. The disk of NGC 4123 has no sign of truncation out to 10 scale lengths, and star-forming regions are found well outside $R_{25}$. A Fabry-Perot $\mathrm{H} \alpha$ velocity field shows velocity jumps of $>100 \mathrm{~km} \mathrm{~s}^{-1}$ at the location of the dust lanes within the bar, indicating shocks in the gas flow. VLA observations yield the velocity field of the H I disk. Axisymmetric mass models yield good fits to the rotation curve outside the bar regionfor disk $I$-band $M / L$ of 2.25 or less, and dark halos with either isothermal or power-law profiles can fit the data well. In a companion paper, we model the full 2-D velocity field, including non-circular motions, to determine the stellar $M / L$ and the mass of the dark halo.
\end{abstract}

Subject headings: galaxies: kinematics and dynamics — galaxies: halos — galaxies: structure — galaxies: individual: NGC 4123

\section{INTRODUCTION}

The rotation speed of gas in a disk galaxy is directly related to its mass, providing a dynamical measure of galaxy mass if the gas is on circular orbits in the disk plane (e.g. Öpik 1922; Roberts 1969; Tully \& Fisher 1977). The rotation curve $V_{c}(R)$ yields the radial profile of the enclosed mass $M(<R)$, modulo the effects of the flattening of the mass distribution. Many galaxies have nearly flat rotation curves, requiring dark mass more extended than the luminous material (e.g. Babcock 1939; Rubin, Ford, \& Thonnard 1978; Bosma 1981).

Unfortunately, even a high quality rotation curve extending well beyond the optical disk does not constrain the relative contributions of dark and visible matter to the centripetal acceleration; such decompositions are almost totally degenerate. For example, van Albada et al. (1985) showed that the rotation curve of the well-observed galaxy NGC 3198, the "poster child" for dark matter (DM) halos, can be fitted equally well with a massive disk and a dark halo significant only in the outer parts of the galaxy, a less massive disk and a more dominant halo, or even just a halo and a zero-mass disk.

It is critical to try to determine the relative masses of disks and halos in galaxies because uncertainty in this quantity impacts almost every area of galaxy formation and dynamics. It hampers our understanding of: the

${ }^{1}$ Visiting Astronomer, Cerro Tololo Inter-American Observatory.

${ }^{2}$ Visiting Astronomer, National Radio Astronomy Observatory. structure and formation process of disk galaxies; the efficiency of assembly of baryons into disks; the Tully-Fisher relation between luminosity and rotation width; instabilities which give rise to spirals and bars, warps, mergers, etc. Furthermore, ignorance of dark halo densities and masses leaves us without a probe of the initial density fluctuation power spectrum on galaxy scales.

One approach to this problem has been to place a lower bound on the mass of the inner DM halo by constructing a "maximum disk" model to fit the rotation curve (Kalnajs 1983; van Albada \& Sancisi 1986). Such a model attributes the highest possible mass-to-light ratio $(M / L)$ to the luminous matter, usually consistent with a non-hollow DM halo. There are a number of indirect dynamical arguments suggesting that the disk mass is in fact close to maximum for most bright galaxies (e.g. van Albada \& Sancisi 1986; Freeman 1992; Debattista \& Sellwood 1998) or nearly so (Athanassoula, Bosma \& Papaioannou 1987; Bosma 1999). Counter-arguments against maximum disks have been advanced by Efstathiou, Lake, \& Negroponte (1982), Bahcall \& Casertano (1985), Bottema (1993, 1997), van der Kruit (1995), Courteau \& Rix (1999) and others. Several recent studies, motivated by cosmological structure formation simulations, have addressed dark halo structure and the relation between dark halo size and galaxy size (e.g. Navarro et al. 1996, Syer \& White 1998, Syer, Mao, \& Mo 1999) and have generally favored strongly sub-maximal disk models.

While models with zero mass disks can fit rotation 
curves, all reasonable models attribute some mass to the luminous component. The disagreement between maximum disk and halo-dominated models such as those of Bottema (1997) is roughly a factor of 2.5 in disk $M / L$. Population synthesis estimates of stellar $M / L$ ratios cannot resolve this discrepancy, because most of the light is emitted by massive stars while most of the mass is in faint stars (Worthey 1994; Charlot, Worthey \& Bressan 1996).

Barred galaxies offer a way to break the disk-halo degeneracy. The elongated bar drives non-circular streaming motions in stars and gas within the bar region. The magnitude of the non-circular motions depends mostly on the mass of the bar and, to a lesser extent, on the pattern speed at which it rotates. The departures from axisymmetry in both light distribution and kinematics can be observed. The (unobservable) dark halo is thought to be mostly "pressure" supported and therefore more rounded than the bar. The stellar bar is therefore the dominant source of non-circular streaming motions, which allow us to estimate its $M / L$, and by extension that of the stellar disk.

In practice, we need extensive observations and modeling to carry out this program. The most easily observable kinematic tracers are optical and radio emission lines from gas in the galaxy disk. Because the gas is not on circular orbits, and we observe only line-of-sight velocities, we cannot infer the full space velocity field of the gas, as we can for an axisymmetric galaxy. Furthermore, gas flow in a barred potential generally gives rise to shocks, which prevent us from directly inverting the observed gas velocities to derive the mass distribution. We therefore need to derive the bar mass by matching fluid models incorporating shocks to the observed velocity field.

In this paper we describe a detailed study of the barred galaxy NGC 4123. We selected this galaxy for its fairly strong bar, a moderate inclination, favorable projection angle of the bar, colors and morphology suggesting substantial $\mathrm{H} \alpha$ emission, and modest size. Because projection of a non-axisymmetric velocity field into the observable line-of-sight velocity depends on its angle to the galaxy major axis (line of nodes), candidates for study must be selected carefully (Pence \& Blackman 1984b; Long 1991). If the bar is aligned with either the major or minor axis of the galaxy disk as it appears on the sky (e.g. NGC 1300, England 1989), it is difficult to separate the circular and non-circular parts of the velocity field after projection, since the orbits or streamlines are elongated in approximately the same direction as the bar (e.g. Athanassoula $1992 \mathrm{~b})$. The projection is most favorable for bars at intermediate position angles - a $45^{\circ}$ angle between the bar and the line of nodes is best.

We require two-dimensional surface photometry in several broad-band filters and a 2-D velocity map over the entire galaxy. "Three-dimensional" imaging spectroscopy is possible with Fabry-Perot interferometers at optical wavelengths, and with aperture-synthesis interferometers at radio wavelengths. These techniques are complementary in angular resolution and field of view, and the spectral lines arise from different phases of the interstellar medium. This galaxy is the first of several barred galaxies we have observed with the Rutgers Imaging Fabry-Perot Interferometer at CTIO (Schommer et al. 1993).

We present surface photometry and velocity maps of

\begin{tabular}{ll}
\hline \hline Parameter & Value \\
\hline Recession velocity & $1327 \mathrm{~km} \mathrm{~s}^{-1}$ \\
Assumed distance & $22.4 \mathrm{Mpc}$ \\
$m_{B}$ (within $\left.3 R_{25}\right)$ & 12.06 \\
$m_{V}$ (within $\left.3 R_{25}\right)$ & 11.45 \\
$m_{I}$ (within $\left.3 R_{25}\right)$ & 10.33 \\
PA & $-33 \pm 2^{\circ}$ \\
inclination & $45 \pm 4{ }^{\circ}$ \\
$L_{I}$ (extinction corrected) & $1.72 \times 10^{10} L_{\odot}$ \\
$V_{\text {rot }}$ & $130 \mathrm{~km} \mathrm{~s}^{-1}$ \\
$21 \mathrm{~cm}$ flux & $63.5 \mathrm{Jy} \mathrm{km} \mathrm{s}^{-1}$ \\
$M_{H I}$ & $7.5 \times 10^{9} \mathrm{M}_{\odot}$ \\
\hline
\end{tabular}

TABLE 1

NGC 4123 - Basic Properties

NGC 4123, from which we construct axisymmetric models. Mass models with a maximum disk $M / L$ of 2.25 and either isothermal or power-law profiles can fit the rotation curve well. In a companion paper (Weiner et al. 2000, hereafter Paper II), we model the full 2-D velocity field to determine the separate masses of the disk and halo.

\section{NGC 4123 - BASIC PROPERTIES}

NGC 4123 is of moderate size and has no pronounced morphological peculiarities. It is an $\mathrm{SB}(\mathrm{r}) \mathrm{c}$ galaxy with total blue magnitude $B_{T}=11.98$, a rotation amplitude of approximately $130 \mathrm{~km} \mathrm{~s}^{-1}$, and a recession velocity of $1327 \mathrm{~km} \mathrm{~s}^{-1}$, from the RC3 catalog (de Vaucouleurs et al. 1991), via NED 3 . We adopt a distance of $22.4 \mathrm{Mpc}$ $(m-M=31.75)$, based on a Hubble constant of 75 $\mathrm{km} \mathrm{s}^{-1} \mathrm{Mpc}^{-1}$ and a correction to the microwave background rest frame, with no Virgocentric correction. Dynamical estimates of $M / L$ vary inversely with the adopted distance $D ; M / L$ values given in this paper are in solar units and are implicitly followed by the factor $h_{75}$ $\left(H_{0} / 75 \mathrm{~km} \mathrm{~s}^{-1} \mathrm{Mpc}^{-1}\right)$. However, estimates of the degree of disk maximality (disk-to-halo ratio) do not depend on adopted distance. The basic properties of NGC 4123 are summarized in Table 1 .

NGC 4123 has a fairly strong bar, but the bar does not totally dominate the disk (unlike e.g. NGC 1097 or NGC 1365; cf. Ondrechen \& van der Hulst 1989; Ondrechen et al. 1989; Lindblad, Lindblad, \& Athanassoula 1996), so it is not an extreme case. The galaxy has a favorable inclination $\left(45^{\circ}\right.$ as determined from $21 \mathrm{~cm}$ observations, see below) and the bar is aligned at approximately $53^{\circ}$ to the line of nodes when deprojected, which is almost ideal for recovering non-circular motions.

NGC 4123 has a companion galaxy, NGC 4116, which lies $14^{\prime}$ southwest of NGC 4123 and at nearly the same systemic velocity, $1312 \mathrm{~km} \mathrm{~s}^{-1}$. NGC 4116 is of $\mathrm{SB}(\mathrm{rs}) \mathrm{dm}$ type and is fainter than NGC 4123, with magnitude $B_{T}=$ 12.41, from the RC3 via NED. At 22.4 Mpc, the separation of the galaxies in the plane of the sky is just $91 \mathrm{kpc}$. However, neither shows much sign of tidal interaction (see Sec-

\footnotetext{
${ }^{3}$ The NASA/IPAC Extragalactic Database (NED) is operated by the Jet Propulsion Laboratory, California Institute of Technology, under contract with the National Aeronautics and Space Administration.
} 
tion 5) and it is possible that the real separation is much greater. The largely undisturbed kinematics of the two galaxies, shown in Taylor et al. (1995) and discussed further below, indicate that any interaction does not visibly affect their internal dynamics. The conclusions we draw from studying the dynamics of the bar region of NGC 4123 are unaffected by the presence of NGC 4116 .

\section{SURFACE PHOTOMETRY}

We require surface photometry in order to estimate the distribution of luminous mass in the galaxy. It is usual to assume that mass is proportional to luminosity; a uniform $M / L$ has the advantage of being the simplest possible assumption, and is generally thought to be reasonable when color variations are small. This is difficult to prove, since the bulk of the mass is in low mass stars while high mass stars emit much more light per unit mass. Furthermore, extinction is particularly variable in barred galaxies, which generally have strong dust lanes within the bar. Such problems are lessened at red and near IR wavelengths, since bluer optical bands are more strongly affected by emission from young massive stars and by spatially variable extinction, although $I$ (and even $K$ ) band observations are still affected by young stars and by dust (de Jong 1996b, Rhoads 1998).

\subsection{Observations}

The initial imaging observations of NGC 4123 were made on the night of 1994 April 1 with the 0.9-meter telescope of Cerro Tololo Interamerican Observatory. We used a Tektronix $1 \mathrm{~K} \times 1 \mathrm{~K} \mathrm{CCD}$ at the $\mathrm{f} / 13.5$ Cassegrain focus, with a pixel scale of $0.39^{\prime \prime}$, and a field of view of 6.6 ${ }^{\prime}$. We observed NGC 4123 in $B V R I$ filters, obtaining 10 minute exposures in $B V R$ and $3 \times 10$ minute exposures in $I$, offset between exposures to improve flatness of the final image. The seeing was $1.2^{\prime \prime}$ in $V R I$, or $130 \mathrm{pc}$ at the adopted distance of $22.4 \mathrm{Mpc}$, and $1.4^{\prime \prime}$ in $B$. We reduced the images with IRAF , subtracting overscan and a bias frame made by a robust combination of many individual biases, and flatfielding with twilight flats in the usual way. Photometric standard stars from the E-regions (Graham 1982) were observed at several times during the night and used to derive extinction coefficients.

The field of view of the CTIO observations is rather small for such a large, nearby galaxy. We reobserved NGC 4123 to obtain a larger field, using the Swope 1-meter telescope at Las Campanas Observatory on the night of 1998 January 25. These observations used the LCO Tek\#5 2K $\mathrm{x} 2 \mathrm{~K} \mathrm{CCD}$ at the $\mathrm{f} / 7$ Cassegrain focus, with a pixel scale of $0.70^{\prime \prime}$ and field of view of $24^{\prime}$. We obtained 10 minute exposures of NGC 4123 in $B V$ filters and $2 \times 10$ minute offset exposures in the $I$ filter. The seeing was approximately $1.6^{\prime \prime}$ in $V I$ and $1.8^{\prime \prime}$ in $B$. The images were again reduced with IRAF, subtracting overscan and bias and flatfielding with twilight flats. Photometric standard stars from Landolt (1992) were observed throughout the night and used to derive extinction coefficients. The photometric calibra-

${ }^{4} \mathrm{CTIO}$ is operated by the Association of Universities for Research in Astronomy, Inc. under a cooperative agreement with the National Science Foundation.

${ }^{5} \mathrm{IRAF}$ is distributed by NOAO, which is operated by AURA Inc., under a cooperative agreement with the National Science Foundation. tions of the CTIO and LCO data are consistent.

The LCO Tek\#5 chip exhibits fringing and illumination gradients in the $I$ filter which cannot be removed with twilight flats, since the night sky spectrum is of a different color and contains strong emission lines. We constructed a supersky image by combining a number of dithered $I$ images from throughout the 3-night run, scaled by the sky brightness, and subtracted this image from the NGC 4123 $I$ image. This process removed both small-scale fringes and large-scale gradients in the image.

\subsection{Morphology}

The inner part of the combined CTIO 0.9-m I image of NGC 4123 is presented in Figure 11. The bar has a semi-major axis of approximately $50^{\prime \prime}$ on the sky, as determined by the transition from boxy to essentially elliptical isophotes, and by the break in the surface brightness profile (see below), or $5.6 \mathrm{kpc}$ when deprojected. The bar has a rather complex shape, with a bright boxy component near the center and a fainter elongated component, which is of constant width or even broadens a little towards the ends; there is some evidence for an isophotal twist between the two components. The complex shape makes it difficult to define a single axis ratio for the bar: the elongated component has an axis ratio of about $6: 1$, while the boxy component has an axis ratio of about 2.5:1.

Other than the central source (discussed below), NGC 4123 has no significant spherical bulge, which makes it particularly suitable for the deprojection method we use in our simulations of the gas flow in this galaxy (see Paper II). Outside the bar region, the disk of NGC 4123 is relatively smooth in $I$ light, with some spiral structure but no sharp features or strong brightness variations.

There is an isophotal twist in the outer regions of the disk. At very large radii the PA and axis ratio of the isophotes are consistent with the PA of the galaxy major axis and the inclination as derived from $21 \mathrm{~cm}$ observations of the H I disk of the galaxy (see section 5). However, at radii less than $\sim 100^{\prime \prime}$, but well outside the bar, the PA of the disk isophotes is intermediate between the PA of the bar and the PA of the kinematic major axis indicated by the $21 \mathrm{~cm}$ observations. A heavily smoothed image shows a faint spiral arm pattern in the outer disk, which is responsible for the isophotal twist. There is no large-scale twist in the kinematic line of nodes in this range of radii in the $\mathrm{H}$ I observations, which indicates that the isophotal twist is not accompanied by a warp (Briggs 1990).

The $B-I$ color map, presented in Figure 2, was constructed from CTIO $B$ and $I$ images convolved to $1.8^{\prime \prime}$ resolution. The dust lanes, conspicuous in this color map, are linear features, offset from the bar major axis towards its leading edge. Dust lanes of this geometry are common in strongly barred galaxies (Sandage 1961; Sandage \& Bedke 1988; Athanassoula 1992b and references therein). Colorcolor plots of pixels within the bar (as in Quillen et al. 1995) show that in the east dust lane, the slopes of $R-I$ and $V-I$ versus $B-I$ are consistent with a dust absorbing screen with $R_{V}=3.1$ (Cardelli, Clayton \& Mathis 1989), confirming that the dust lane in the east half is near to us. In the west dust lane the slopes are shallower, so that the dust lane is on the far side of the bar, as expected.

The small bright nucleus at the center of the galaxy stands out as the central blue spot. It is barely resolved 

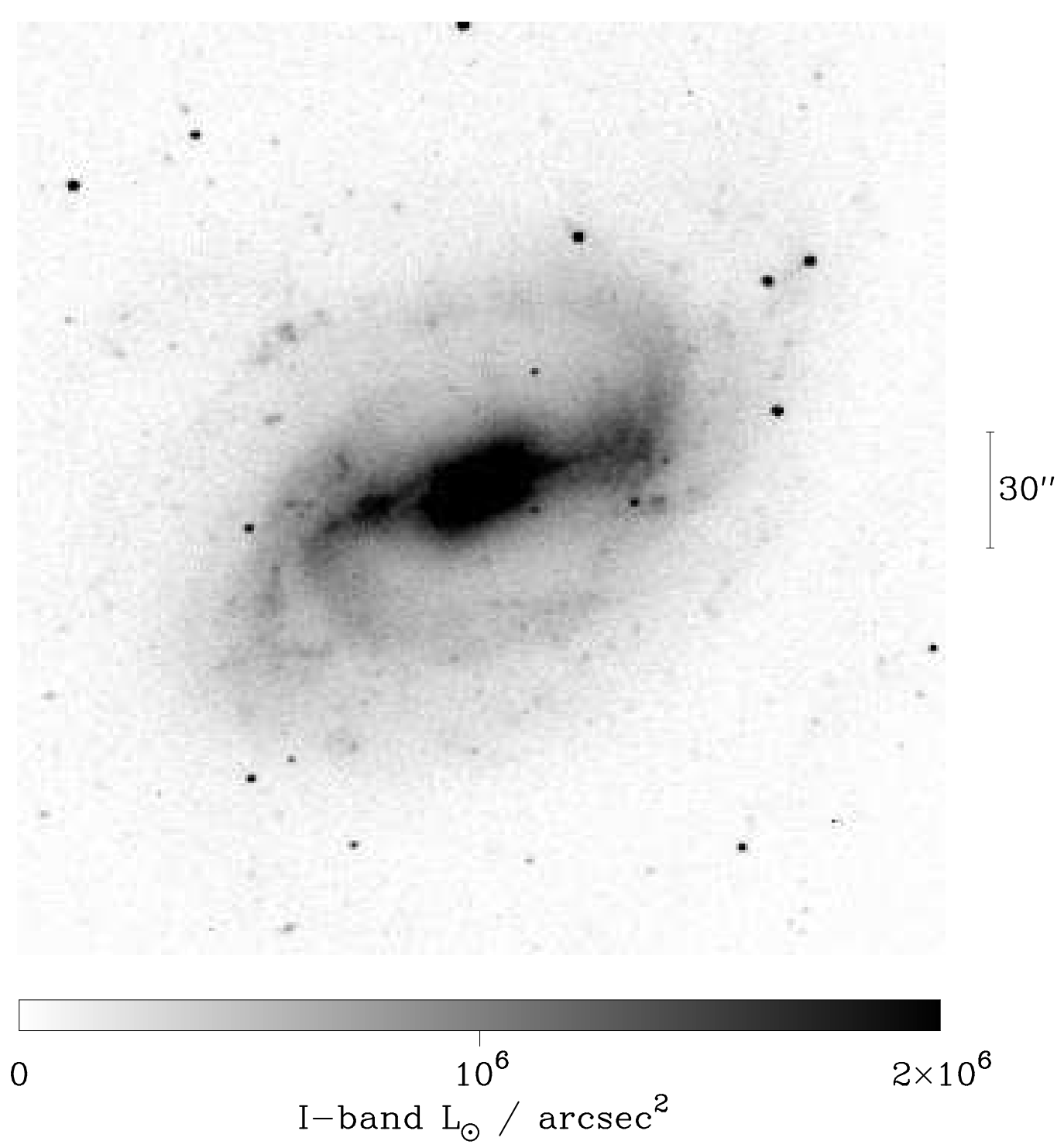

FiG. 1.- NGC 4123, $I$ image.

The inner $240^{\prime \prime}$ square region of the CTIO 0.9-m I-band image of NGC 4123, on a linear scale. North is up and west is to the right. The major axis of the disk is $33^{\circ}$ west of north. [Resolution degraded by a factor of 2 for preprint version.]

by these observations. Its color, $B-I=1.4$, is quite blue compared to its surroundings $(B-I=2.2)$, and its luminosity in the $I$ band is $1.8 \times 10^{8} L_{\odot}$. The center of NGC 4123 appears in lists of known emission line and UVexcess sources as UM 477 (MacAlpine \& Williams 1981) and Markarian 1466, suggesting that it is a nuclear starburst. It is likely, therefore, that the $M / L$ of the nucleus is lower than the overall $M / L$ of the stellar disk, a point we return to in the models of Paper II.

\subsection{Surface brightness profiles}

We constructed axisymmetrized surface brightness profiles of NGC 4123 from the Las Campanas BVI data. The $21^{\prime}$ field allows us to extend the surface brightness profiles to large galactic radius.

We first masked approximately 1100 stars and faint galaxies and a small number of cosmic rays out of the image, using large masks around the stars to exclude the faint halo of the stellar PSF. The surface brightness profiles are then constructed by dividing the image into elliptical annuli, $1.7^{\prime \prime}$ wide along the major axis, with the disk inclination of $45^{\circ}$ and position angle of $-33^{\circ}$ as derived from the $21 \mathrm{~cm} \mathrm{H}$ I observations (see below). In each annulus, we compute the trimmed mean with a 2 -sigma clipping. We determined the sky level by computing the biweight, a robust estimate of the mode (Beers, Flynn \& Gebhardt 1990), of all pixels within an elliptical annulus of the same inclination and PA, an inner major axis radius of $551^{\prime \prime}(60 \mathrm{kpc})$ and outer major axis radius of $593^{\prime \prime}$ (65 $\mathrm{kpc}$ ). The error estimate is computed directly from the dispersion of the pixel values in each annulus, excluding clipped pixels, with the statistical error of the sky value added in quadrature.

The axisymmetrized $B, V$, and $I$ surface brightness profiles of NGC 4123, and the color profiles, are shown in Figure 3. We have not included a correction for internal 

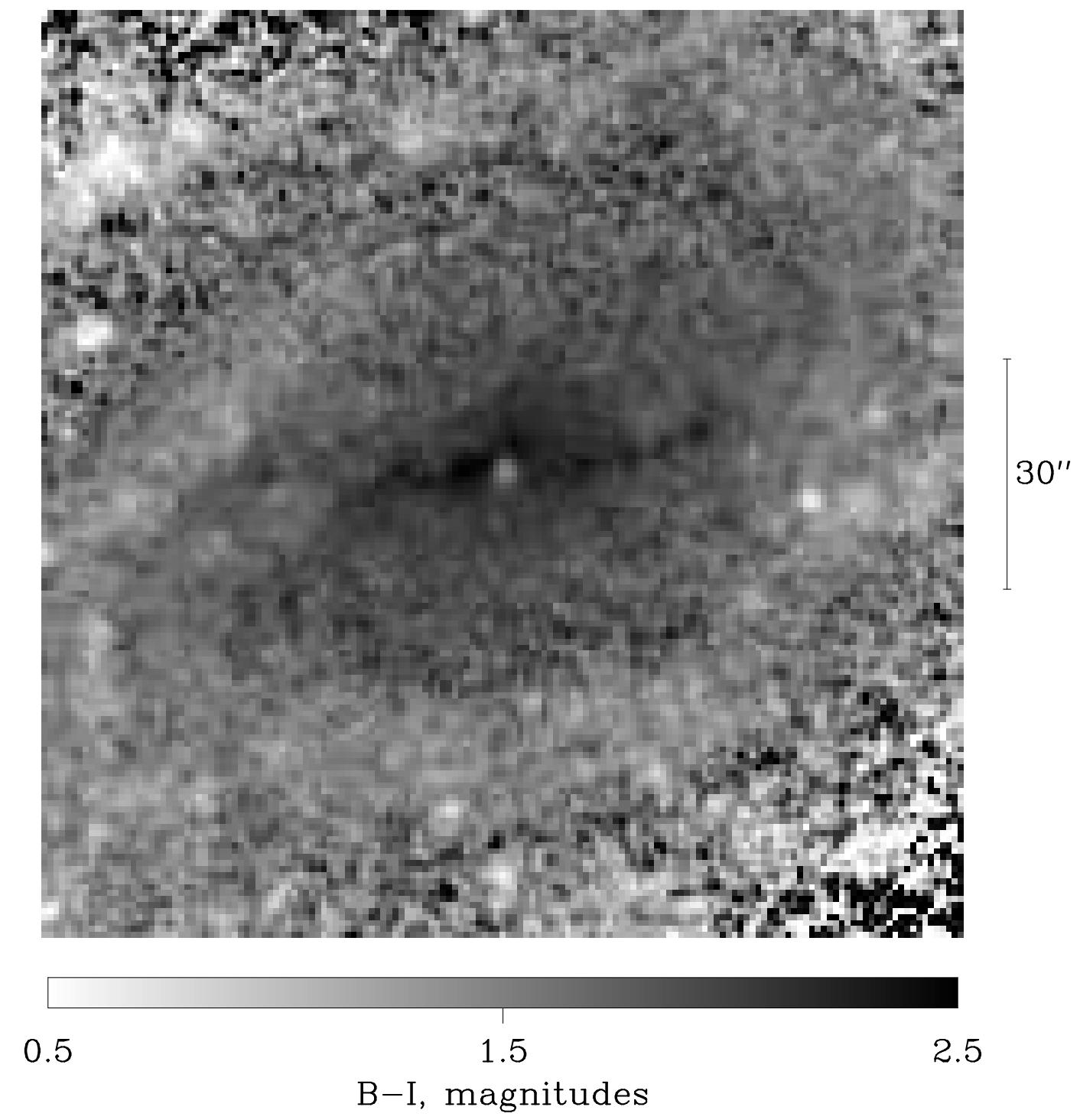

Fig. 2.- NGC 4123, $B-I$ color map

The inner $120^{\prime \prime}$ square region of the CTIO $B-I$ color map of NGC 4123. North is up and west is to the right. Darker colors are redder. The dust lanes in the bar are apparent as the dark lines running east and west of the nucleus. The nucleus of the galaxy is much bluer than its surroundings. The blue spots at the west end of the bar are foreground stars. [Resolution degraded by a factor of 2 for preprint version.]

extinction in these plots, but we have corrected for geometric inclination to obtain a face-on surface brightness:

$$
\mu_{\text {corr }}=\mu_{\mathrm{obs}}+2.5 \log (\mathrm{b} / \mathrm{a}),
$$

as appropriate for a transparent galaxy. At an inclination of $45^{\circ}$ this correction is $+0.38 \mathrm{mag} \operatorname{arcsec}^{-2}$. The shape of the profiles, the colors, and the color gradient are typical of SBbc galaxies.

There is a substantial color gradient, $\sim 0.4 \mathrm{mag}$, inside the bar radius, but this does not imply an equally large color gradient in the old stellar population. Inside the bar, the strong dust lanes produce reddening, while outside the bar, many $\mathrm{H}$ II regions with very blue colors are present in the spiral arms. We measured mean colors in several regions in the bar and disk in and out of dust lanes and $\mathrm{H}$ II regions. The results are given in Table 2. When obvious dust lanes and $\mathrm{H}$ II regions are excluded, there is

\begin{tabular}{ll}
\hline \hline Region & $B-I$ (mag) \\
\hline Dust lane in bar & 2.3 \\
Bar/lens outside dust lane & 2.0 \\
Disk, interarm region & 1.8 \\
Disk, spiral arms & 1.5 \\
\hline
\end{tabular}

TABLE 2

NGC 4123 - MeAn Colors

only an 0.2 mag difference in $B-I$ between the bar and disk, so there is no evidence for a significant difference in stellar population. Since unresolved dust lanes and knots of young stars may be present, the actual color difference could be smaller.

In our subsequent use of the $I$ data to fit the rotation 
curve and model the potential, we apply a -0.15 mag overall correction for internal extinction in the $I$ band, calculated from the $A_{\text {int }}=-1.0 \log (b / a)$ prescription given by Giovanelli et al. (1994), which Palunas (1996) found to be reliable for similar $I$-band data. Galactic extinction $A_{\text {ext }}$ towards NGC 4123 is negligible (Burstein \& Heiles 1982).

We did not attempt to correct the $I$ image for differential extinction within the galaxy. This procedure requires extensive multicolor data, including near-IR imaging, and detailed modeling of radiative transfer (see Byun et al. 1994; Regan et al. 1995; de Jong 1996b). To estimate the extinction in the east (stronger) dust lane, we selected a region of the bar where the dust lane appeared strong in $B$ and took a cut through the data perpendicular to the dust lane. Assuming the unextincted bar light profile is symmetric when reflected about the bar major axis, we estimate the differential extinction to be $\sim 25 \%$ ( $0.3 \mathrm{mag}$ ) in $B$ and $\sim 10 \%(0.1 \mathrm{mag})$ in $I$. Alternatively, for the dust lane reddening of $E_{B-I}=0.3$, an $R_{V}=3.1$ extinction law (Cardelli, Clayton \& Mathis 1989), and an absorbing dust screen, the dust lane extinction in $I$ is $0.17 \mathrm{mag}$. Since the dust lanes cover only a small part of the bar, the amount of $I$ light lost to extinction is just a few percent.

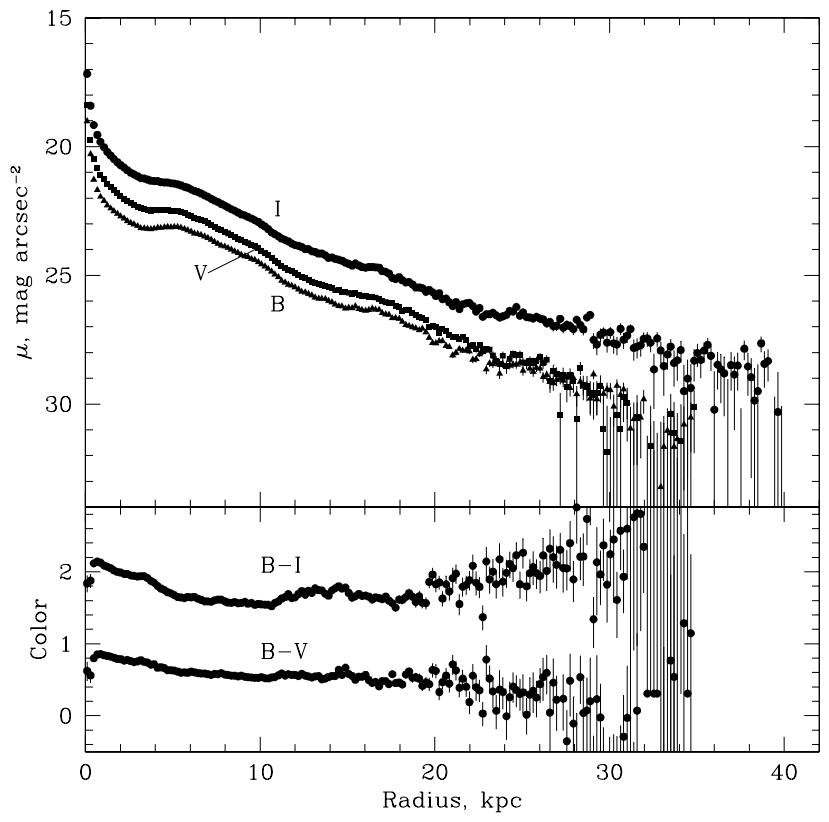

FIG. 3.- $B V I$ surface brightness and color profiles of NGC 4123 Upper panel: The axisymmetrized LCO $B, V, I$-band surface brightness profiles of NGC 4123, in magnitudes $\operatorname{arcsec}^{-2}$. The profiles have been corrected for inclination but not internal extinction. $B, V, I$ are triangles, squares, and circles respectively. The $I$-band profile is plotted to $40 \mathrm{kpc}$ while $B$ and $V$ are plotted to $35 \mathrm{kpc}$.

Lower panel: The axisymmetrized $B-V$ and $B-I$ color profiles of NGC 4123 , in magnitudes $\operatorname{arcsec}^{-2}$. The profiles have not been adjusted for internal reddening.

The luminosity profile has a strong peak at the center, a shoulder in the bar region, and an approximately exponential decrease outside the bar, though the slope changes somewhat. The radius at which the profile breaks to exponential, $\sim 50^{\prime \prime}$, or $5.5 \mathrm{kpc}$, is the same as the bar length. This type of surface brightness profile, with flat and exponential regions, was designated type II by Freeman (1970), and is fairly common in both axisymmetric and barred galaxies; see for example the sample of Palunas \& Williams (2000).

Table 3 summarizes overall photometric parameters of NGC 4123 from the LCO observations. The nominal optical radius of the galaxy $\left(R_{25}\right.$ in $\left.B\right)$ is $11.1 \mathrm{kpc} ; R_{23.5}$ in $I$ is $11.3 \mathrm{kpc}$. The exponential disk scale lengths given in Table 3 were determined from least-squares fits between 6 and $10 \mathrm{kpc}$, where the data are well described by an exponential. The inward extrapolated central surface brightness in I of $19.59 \mathrm{mag}$ (19.44 mag when corrected for extinction) makes this galaxy relatively high surface brightness, but not extremely so (de Jong 1996a; Palunas 1996). The total $I$ magnitude within $3 R_{25}$ is 10.33 ; the absolute magnitude, corrected for internal extinction, is $M_{I}=-21.57$ and luminosity $L_{I}=1.72 \times 10^{10} L_{\odot}$. The absolute $B$ magnitude, uncorrected for extinction, is $M_{B}=-19.7$, a luminosity of $0.7 L_{*}$.

\subsection{Outer disk profiles}

We have been able to trace the surface brightness profiles to quite large radii, beyond $2 R_{25}$, even to $3 R_{25}$ for $I$ (9 magnitudes below the sky). At very large radii the profiles become uncertain due to a combination of photon noise and errors in the sky level. Beyond $20 \mathrm{kpc}$, the color profiles are probably not reliable - in particular, the reddening trend seen in $B-I$ but not in $B-V$ could be due to a systematic error in the $I$ sky level. Some small-scale features in the profiles are certainly real, such as the red bump in $B-I$ at $12-14 \mathrm{kpc}$ and the bump in all three filters at $16 \mathrm{kpc}$, which both correspond to faint spiral arms. There is also a spiral arm at $\sim 24 \mathrm{kpc}$.

The surface brightness profiles show no sign of reaching a plateau before they disappear into the noise, at $R \sim 30 \mathrm{kpc}$ for $B$ and $V$, and $35 \mathrm{kpc}$ for $I$, so the large extent of the galaxy is not due to undersubtraction of the sky. We constructed an extended PSF from observations of bright stars to measure the contribution of scattered light, which is negligible. Scattered light outside a sharply truncated disk would produce a surface brightness profile with an observed drop of 4 magnitudes at the disk edge, which is clearly ruled out.

In all three filters, the galaxy is easily visible to $R \sim$ $25 \mathrm{kpc}$ when displayed at high contrast, and to greater extent when the images are smoothed with a median filter. Spiral arms are traceable to $R \sim 25 \mathrm{kpc}$; the arms are strongest in the $B$ filter, while in the $I$ filter the extended galaxy light is more smoothly distributed than in $B$ and $V$.

There is no evidence in the data that the disk is truncated at large radius, in sharp contrast with results that suggest disk galaxies are generally truncated at 3 to 5 scale lengths (e.g. van der Kruit \& Searle 1981a,b, 1982; van der Kruit 1989; Barteldrees \& Dettmar 1994; Morrison, Boroson \& Harding 1994). The SB profiles of NGC 4123 are traceable twice as far, to 10 scale lengths in the $I$ filter. At least $20 \%$ of the galaxy luminosity is outside $R_{25}$. One other galaxy, NGC 5383, definitely does not have a truncated disk (Barton \& Thompson 1997).

The origin of this extended stellar disk is uncertain. It has been suggested that truncated disks occur because a stability condition cuts off star formation below a critical gas surface density (Kennicutt 1989; Sellwood \& Balbus 
Weiner et al.

\begin{tabular}{|c|c|c|c|c|c|c|c|}
\hline \multirow[t]{2}{*}{ Filter } & \multirow{2}{*}{$\begin{array}{c}\text { Night sky } \\
\text { brightness } \\
\text { mag/ } \square^{\prime \prime}\end{array}$} & \multirow{2}{*}{$\begin{array}{c}2 \sigma / \text { pixel } \\
\mu_{\text {lim }} \\
\mathrm{mag} / \square^{\prime \prime}\end{array}$} & \multicolumn{2}{|c|}{ exp disk } & \multicolumn{3}{|c|}{ magnitude within } \\
\hline & & & $\begin{array}{c}\mu_{0} \\
\mathrm{mag} / \square^{\prime \prime}\end{array}$ & $\begin{array}{l}\text { scalelength } \\
\text { kpc }\end{array}$ & $R_{25}$ & $\begin{array}{c}2 R_{25} \\
\text { mag }\end{array}$ & $3 R_{25}$ \\
\hline $\mathrm{B}$ & 22.35 & 25.11 & 21.38 & 3.46 & 12.29 & 12.09 & 12.06 \\
\hline $\mathrm{V}$ & 21.52 & 24.64 & 20.65 & 3.25 & 11.65 & 11.46 & 11.45 \\
\hline I & 19.43 & 23.38 & 19.59 & 3.20 & 10.55 & 10.36 & 10.33 \\
\hline
\end{tabular}

TABLE 3

NGC 4123 - LCO Photometric Data

Note. - The central surface brightness $\mu_{0}$ in column 4 has been corrected for inclination, but not for internal extinction.

1999), and it is possible that stars from the inner disk could be scattered to large radius by a bar-like perturbation (Schwarz 1984). However, NGC 4123 has faint spiral arms extending to $\sim 25 \mathrm{kpc}$, with small blue knots that are likely sites of massive star formation. These star-forming regions are similar to the $\mathrm{H}$ II regions in faint spiral arms that Ferguson et al. (1998) detected to $2 R_{25}$ and beyond, in deep $\mathrm{H} \alpha$ imaging of three late-type spirals. Thus at least some of the extended disk of NGC 4123 formed at large radius, although massive star formation in the outer disk appears to be confined to spiral arms, as discussed by Ferguson et al. (1998). The blue knots have luminosities from $M_{B}=-12.5$ to at least 2 magnitudes fainter, and mean colors $B-V \sim 0.1, V-I \sim 0.0$. The very blue colors indicate that the knots are younger than $10^{7} \mathrm{yr}$ (Bruzual \& Charlot 1993). The largest knot has $L_{B}=10^{7} L_{\odot}$, suggesting $M \sim 2 \times 10^{5} \mathrm{M}_{\odot}$. The star formation rate implied outside $R_{25}$ is consistent with the range found by Ferguson et al. (1998).

\section{FABRY-PEROT IMAGING SPECTROSCOPY}

\subsection{Observations}

We observed NGC 4123 in $\mathrm{H} \alpha$ emission with the Rutgers Imaging Fabry-Perot Interferometer to obtain a $2-\mathrm{D}$ velocity field with high spatial resolution. The observations were made at the Cassegrain focus of the CTIO Blanco 4-meter telescope, on the night of 1994 April 3. We used a Tektronix $1 \mathrm{~K} \times 1 \mathrm{~K}$ CCD detector, with $0.36^{\prime \prime}$ pixels and a field of view of $160^{\prime \prime}$. The Fabry-Perot etalon has spectral resolution of $2.5 \AA$ at $\mathrm{H} \alpha$, yielding a velocity resolution of $\mathrm{FWHM} \simeq 150 \mathrm{~km} \mathrm{~s}^{-1}$. The instrumental spectral profile is very well approximated by a Voigt function, with Gaus$\operatorname{sian} \sigma_{G}=26 \mathrm{~km} \mathrm{~s}^{-1}$ and Lorentzian $\sigma_{L}=33 \mathrm{~km} \mathrm{~s}^{-1}$. The free spectral range of this etalon is $85 \AA$ at this wavelength, and a blocking filter of $75 \AA$ FWHM ensured that only one order was transmitted.

The Fabry-Perot instrument provides a normal spatial image of the sky, but with a very narrow, tunable, bandpass. The image is not monochromatic, but varies quadratically with radius from the optical axis of the instrument near the center of the image, with a $6 \AA$ gradient. There are two temporal variations in the instrument: the wavelength zeropoint of the etalon varies due to temporal drift of the control electronics, and the position of the optical axis changes due to spectrograph flexure. In practice, these complications cause little difficulty; we take hourly calibration exposures while observing to monitor the drifts, and the data analysis procedures account for them and for the radial wavelength gradient.

The observations of NGC 4123 are 18 10-minute exposures spanning a range of $20 \AA$ in steps of approximately $1.2 \AA\left(54 \mathrm{~km} \mathrm{~s}^{-1}\right)$. The images were processed using IRAF, and each flatfielded with a dome flat taken at the appropriate wavelength. The images were then cleaned of cosmic ray events, shifted into spatial register, convolved to a common PSF of $1.5^{\prime \prime}$ FWHM, and sky subtracted to construct a data cube. At each pixel in the image we smoothed the data cube spatially with a small Gaussian kernel of $0.6^{\prime \prime}$ FWHM, and then fitted a Voigt function along the spectral axis. The fits yielded maps of the velocity, line strength, continuum level, intrinsic line width, and estimates of the uncertainties in these quantities. Points with intensities below a relatively small threshold or with velocity uncertainties greater than $12 \mathrm{~km} \mathrm{~s}^{-1}$ are deleted from the maps.

The velocity map of NGC 4123 produced from the Fabry-Perot observations is presented in Figure 4. The orientation is the same as that of Figure 1, although the scale is different. The colormap indicates heliocentric velocity; the approaching side of the galaxy is blue, and the receding side is red. The diagonal tick marks indicate the minor axis of the disk. Assuming that the spiral arms are trailing, the northeast side of the galaxy is the side closer to us. The $160^{\prime \prime}$ field of view does not extend much outside the bar region. The velocity map from $21-\mathrm{cm}$ observations, presented in section 5, extends to much greater galactic radius.

\subsection{The streaming motions in the bar}

The non-axisymmetric streaming motions associated with the bar can be seen in Figure 4. In an axisymmetric galaxy, the contour of the systemic velocity would run straight down the minor axis, dividing the map into approaching and receding sides. In NGC 4123, the gas streamlines elongated along the bar cause the isovelocity contours to twist and run more closely along the bar, producing the S-shaped isovels characteristic of barred galaxies (Bosma 1981). To the east and northeast of the galaxy center, there are receding velocities (yellow in the colormap of Figure (4) in the bar region that are on the approaching side of the minor axis, and vice versa to the west.

Furthermore, the isovelocity contours are not straight along the bar, but are offset from the bar major axis. The 


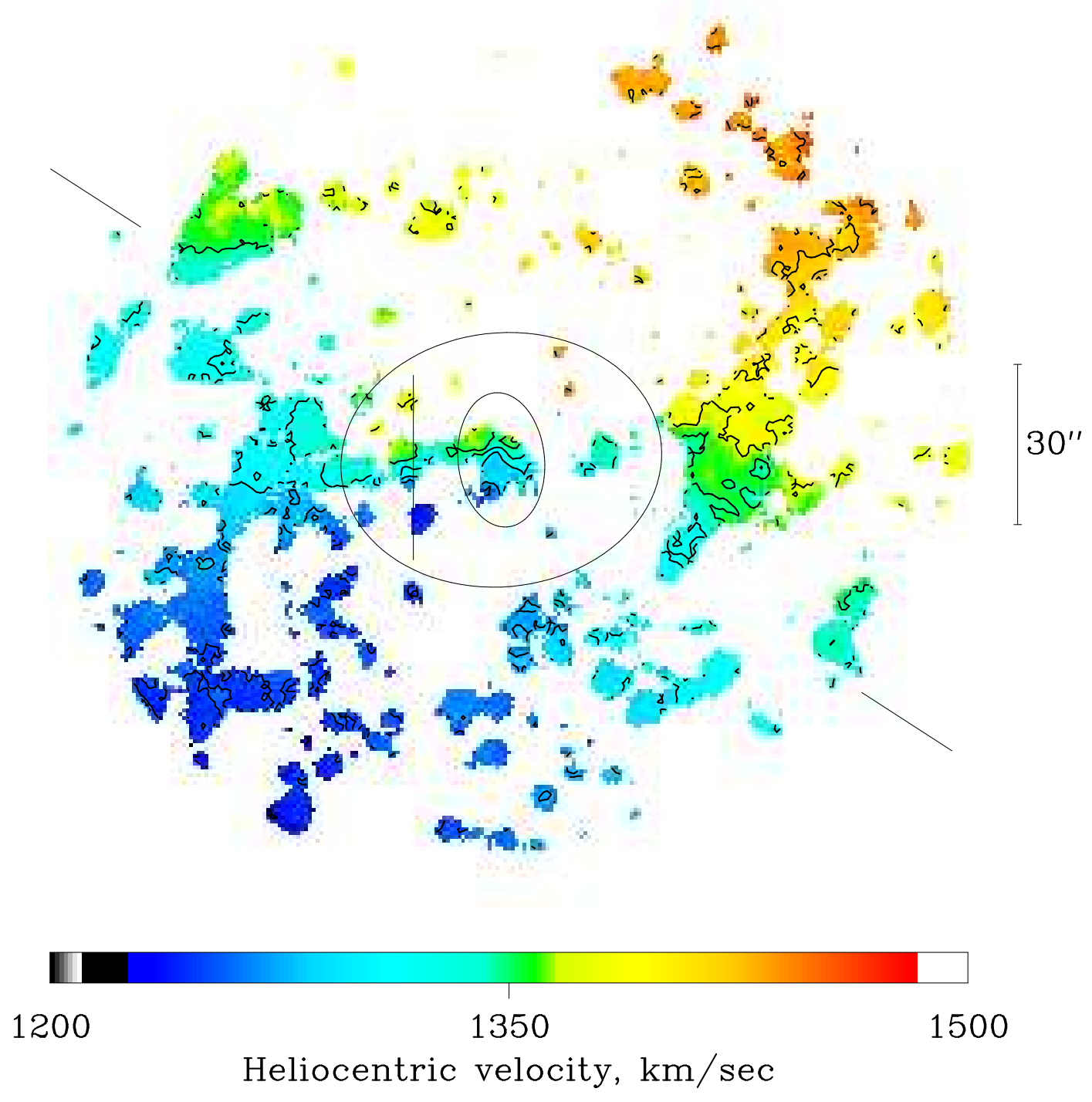

FIG. 4.- The radial velocity field of the bar region of NGC 4123

Velocity map of the inner regions of NGC 4123, from Fabry-Perot observations of the H $\alpha$ emission line. North is up and west is to the right. The area shown is $170^{\prime \prime}$ diameter, smaller than that of Figure 1. Velocity contours ar plotted over the colormap at intervals of $25 \mathrm{~km} \mathrm{~s}{ }^{-1}$. The vertical line to the east of the center indicates the cut through the data plotted in Figure 5 and in Paper II; the data between the two ellipses are used in the goodness-of-fit tests in Paper II. The diagonal lines to NE and SW indicate the minor axis of the disk.

isovels twist as they pass through the center of the bar. For example, the contour of systemic velocity, blue-green in the color map of Figure 1 , is offset toward north on the east side of the bar (left of center in Figure A), makes a jog as it passes through the center, and is offset toward the south on the west side of the bar (right of center in Figure 1). The regions of strong $\mathrm{H} \alpha$ emission in the bar are in similarly offset straight lines. There is also a sharp jump in velocity across the bar, i.e. parallel to the minor axis of the bar. These features suggest the presence of shocks in the gas flow pattern, at the location of the dust lanes in the bar.

Dust lanes are presumably caused by a build-up of gas at shocks in the gas flow, as first suggested by Prendergast (1966) and by many subsequent authors (e.g. van Albada \& Sanders 1982; Prendergast 1983; Athanassoula 1992b; but cf. Beck et al. 1999). These shocks are thought to be caused by the non-circular gas motions along the bar: gas falling down along the bar potential reaches high velocities, and as the gas climbs away from the center, up the potential, it decelerates. Eventually a pile-up occurs and the shock is formed. Velocity jumps associated with bar dust lanes have been observed, in e.g. NGC 5383 (Duval \& Athanassoula 1983), NGC 6221 (Pence \& Blackman 1984a), NGC 1365 (Lindblad et al. 1996a), and NGC 1530 (Regan, Vogel \& Teuben 1997).

The regions of strong $\mathrm{H} \alpha$ emission aligned with the dust lanes in NGC 4123 suggest that compression of the gas by the shocks triggers star formation. The offset of the dust lanes and the associated jog of the velocity contours as they pass through the galaxy center are evidence for an inner Lindblad resonance (ILR) at the bar center (Athanassoula 1992a,b). An ILR can be inferred when some stellar orbits near the center are elongated perpendicular to 
the bar, rather than along it; the gas streamlines twist to follow these stellar orbits (e.g. Figure 2 of Athanassoula $1992 b$ ). This twist creates the jog in the velocity contours and the offset in the shocks along the bar.

The sharp jump in velocity across the bar of NGC 4123, plotted in the upper panel of Figure 5 , is strong kinematic evidence that there are indeed shocks in the bar. The radial velocity in the $\mathrm{H}$ II regions about $20^{\prime \prime}$ east of the galaxy center changes by $80 \mathrm{~km} \mathrm{~s}^{-1}$ in just $4^{\prime \prime}$. The jump is $115 \mathrm{~km} \mathrm{~s}^{-1}$ when corrected for the galaxy inclination - and this is just in the line-of-sight component of the velocity; the absolute velocity jump must be larger. There are also large velocity gradients in the galactic nucleus, inside the ILR, typically $40-50 \mathrm{~km} \mathrm{~s}^{-1}$ in observed velocity (55-70 $\mathrm{km} \mathrm{s}^{-1}$ after inclination correction) across just $2^{\prime \prime}$. This angular size is barely resolved by these observations and the actual gradient is likely to be even steeper. The velocity gradient shown in Figure 5 is at the same location as the dust lane, confirming that the shock and dust lane are associated.

Because the shocks are closely linked to the non-circular gas motions, their properties are dependent on the mass of the bar, which controls the ellipticity of the potential. For this reason, the strength and location of the shocks will prove crucial for measuring the $M / L$ and pattern speed, discussed in Paper II.

There is $\mathrm{H} \alpha$ emission at distances from the galaxy's center of less than the bar radius, but not within the bar itself - for example, the small $\mathrm{H}$ II regions some $20^{\prime \prime}$ northwest of the galaxy center, and the large $\mathrm{H}$ II region $20^{\prime \prime}$ southeast of the center. These regions have large velocities relative to systemic, shown in Figure 5, and provide useful data points for constraining the models of Paper II.

Outside the bar radius, the non-axisymmetric motions fall off rather quickly and the contour of systemic velocity becomes more closely aligned with the minor axis. There are spiral arms with many $\mathrm{H}$ II regions visible to the northwest and southeast, but the velocity perturbations associated with them are much smaller than those in the bar. The limited coverage and field of view of the $\mathrm{H} \alpha$ velocity map makes the global axisymmetric velocity field more difficult to pick out; the $21 \mathrm{~cm} \mathrm{H}$ I velocity map, presented below, shows it well.

\section{RADIO INTERFEROMETRY}

We have used the VLA to image the neutral hydrogen in NGC 4123. The $\mathrm{H}$ I data are complementary to the $\mathrm{H} \alpha$ data. The higher angular resolution of the Fabry-Perot data can trace the kinematics within the bar, while the VLA data can image diffuse $\mathrm{H}$ I and trace the rotation curve far beyond the nominal optical radius $R_{25}$.

\subsection{Observations}

We observed NGC 4123 in the H I $21 \mathrm{~cm}$ line with the $3 \mathrm{~km}(\mathrm{C})$ and $1 \mathrm{~km}(\mathrm{D})$ array of the Very Large Array (VLA) of the National Radio Astronomy Observatory for 8 and 2 hours in December 1994 and April 1995 respectively. This combination gives an angular resolution of $\sim 15^{\prime \prime}$, and provided 35 independent beams along the galaxy major axis, with sensitivity to smooth structures

6 The NRAO is operated by Associated Universities, Inc., under a cooperative agreement with the National Science Foundation.

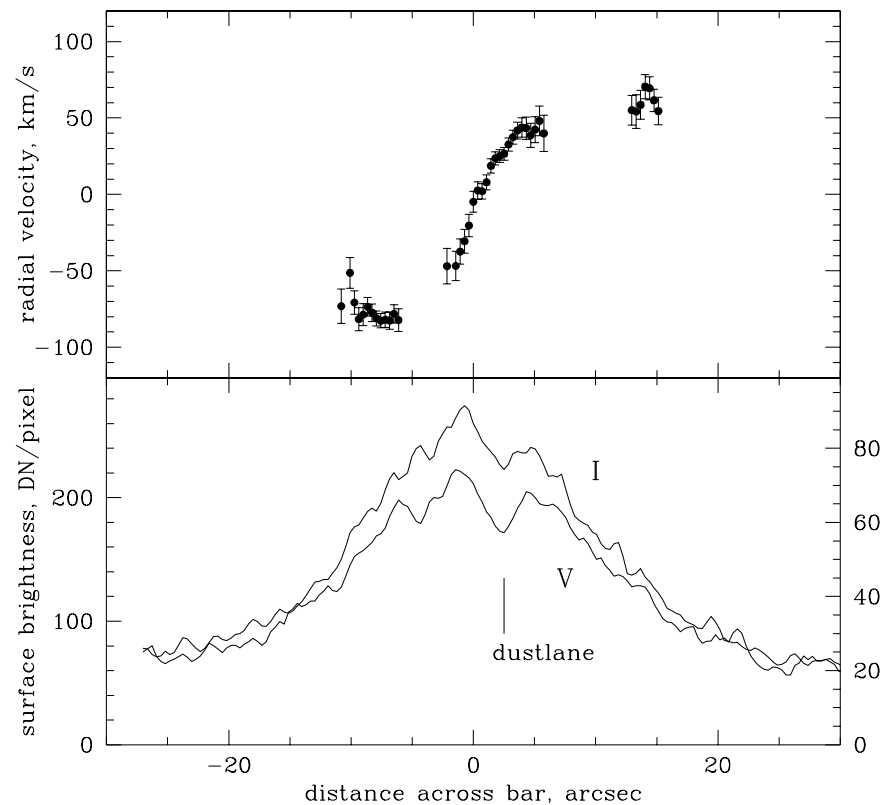

FIG. 5.- Radial velocity and surface brightness profiles crossing the bar

The top panel shows line-of- ight velocity for the north-south line across the bar indicated in Figure 4. South is at negative $x$; the direction of rotation of the galaxy is from left to right. The bottom panel shows $V$ and $I$ surface brightness profiles along the same line. The surface brightness profiles have been sky-subtracted and smoothed with a 3 pixel $\left(1.1^{\prime \prime}\right)$ boxcar filter. The units are linear (counts/pixel) and the $V$ profile has been scaled up by a factor of 3 . The position of the prominent dustlane is indicated.

up to $12^{\prime}$. We used 63 channels with a channel width of $10.5 \mathrm{~km} \mathrm{~s}^{-1}$. Standard AIPS calibration procedures were used and the UV data of the two arrays combined. We subtracted the continuum in the $u-v$ domain using UVLIN, using 19 line-free channels.

We imaged the data with the AIPS task IMAGR using robust weighting (Briggs 1995), yielding a sensitivity almost equal to natural weighting and a resolution almost equal to uniform weighting. An elliptical Gaussian fit to the beam yielded a beam shape of $19.1^{\prime \prime} \times 16.1^{\prime \prime}$ FWHM with a position angle of $56.5^{\circ}$. The rms noise in line-free channels after continuum subtraction is $0.38 \mathrm{mJy} /$ beam. We cleaned the images using the CLEAN algorithm to a flux limit of $0.19 \mathrm{mJy} / \mathrm{beam}$, and made images of total H I intensity, the intensity weighted velocity field, and the velocity dispersion with the AIPS task MOMNT. The total $21 \mathrm{~cm}$ flux observed is $63.5 \mathrm{Jy} \mathrm{km} \mathrm{s}{ }^{-1}$, which recovers all the single-dish flux as tabulated in Huchtmeier \& Richter (1989).

\subsection{Discussion}

The VLA $21 \mathrm{~cm}$ intensity and velocity field shown in the left panel of Figure 6 reveals that NGC 4123 has a large H I disk of $11^{\prime} \times 7.5^{\prime}$, and a regular velocity field. The highest $\mathrm{H}$ I column densities occur in a ring where the surface brightness is mostly above $10^{21} \mathrm{~cm}^{-2}$. The ring's radius is some $70^{\prime \prime}$, or $7.6 \mathrm{kpc}$, placing it somewhat outside the bar. A loose spiral pattern is also visible outside the ring. Inside the ring, the surface brightness decreases toward the center, from $5 \times 10^{20} \mathrm{~cm}^{-2}$ to a low of $4 \times 10^{19} \mathrm{~cm}^{-2}$ at 


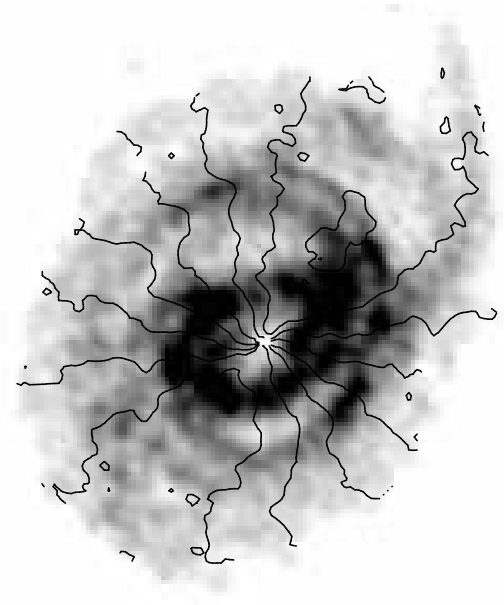

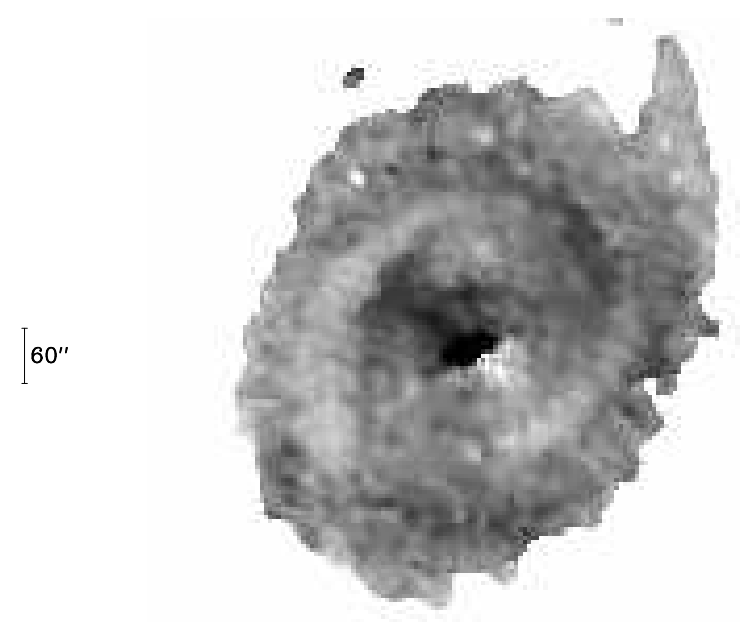

60
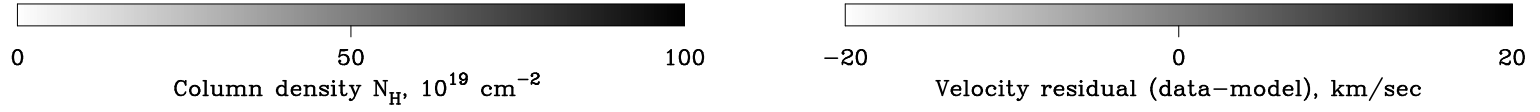

FIG. 6. $-21 \mathrm{~cm}$ emission, velocity field, and residual velocity

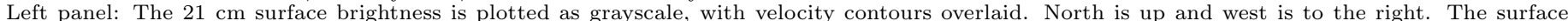

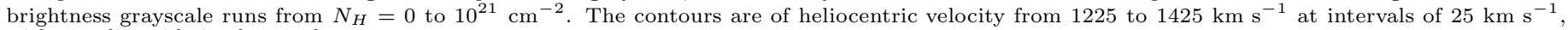
with receding side in the northwest.

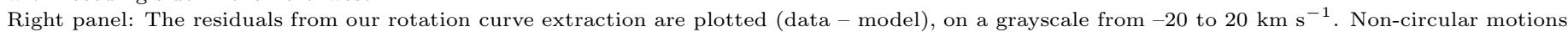
are seen in the bar and in the outer spiral pattern.

the center. The hole in the center of the gas distribution is frequently seen in barred galaxies; e.g. NGC 1300 (England 1989) and NGC 1398 (Moore \& Gottesman 1995). It is presumably caused by the bar, which sweeps gas inside its corotation radius from the bar region towards the galaxy center, as seen in fluid-dynamical simulations (e.g. Athanassoula 1992b; Piner et al. 1995; Weiner \& Sellwood 1999; Paper II).

The overall velocity field in Figure 6 is quite regular. The effect of the bar is visible only inside a radius of $\sim 30^{\prime \prime}$; the contours close to the systemic velocity in this region are twisted to run more east-west, along the bar, rather than along the minor axis of the galaxy.

In the outer parts of the H I disk, velocity perturbations due to the spiral arms are visible as kinks in the velocity contours. These are particularly noticeable in the velocity contours about $160^{\prime \prime}$ northeast of the galaxy center. The contours bow to the northwest as they cross the spiral arm. The effect is of order $10-20 \mathrm{~km} \mathrm{~s}^{-1}$ in line-of-sight velocity. Similar spiral-arm-related kinks of lesser magnitude are visible in other regions of the velocity field.

The only other unusual features in the $\mathrm{H}$ I distribution are the low-column-density arm or extension on the northwest side of the disk, and the even lower column-density fragments of $\mathrm{H} \mathrm{I}$ emission at the north of the disk. The shape of these features suggests that they could be due to some kind of tidal interaction, although because they are close to the detection limit, the "fragments" may not be as isolated as they appear. In any case, the kinematics of the arm and fragments are completely consistent with unperturbed circular rotation. There is no obvious companion very close to the arm, either in the Palomar Sky Survey, the Digitized Sky Survey, our BVI imaging, or the
$21 \mathrm{~cm}$ observations. As mentioned in the introduction, the galaxy NGC 4116 is just $14^{\prime}$ to the southwest of NGC 4123 and is also visible in our VLA observations. We included it in the imaging and CLEANing process and mapped its H I emission and velocity. Figure 7 shows the total H I image and velocity field of both galaxies, corrected for the primary beam response. NGC 4116 has an $\mathrm{H}$ I disk just over half the angular size of the H I disk of NGC 4123, and kinematics consistent with circular rotation and a slowly rising rotation curve.

Tides from NGC 4116 could be responsible for the arm of NGC 4123, but if this is the explanation, it is curious that the counter-arm pointing away from the companion is the more pronounced; furthermore, the larger NGC 4123 does not appear to have distorted the smaller galaxy. The kinematics of the arm can be modeled in circular rotation with the rest of the H I disk of NGC 4123, as described below. Moreover, there is no sign that NGC 4116 has any significant influence on the internal dynamics of NGC 4123 .

\subsection{The rotation curve of $N G C 4123$}

We derived a rotation curve from the $\mathrm{H}$ I data for NGC 4123 using a program written by Palunas (1996) which models the galaxy as a circular, differentially rotating flat disk, and assumes no a priori form for the shape of the rotation curve. The program fits the velocity data in a series of elliptical annuli by adjusting several parameters: central position $x_{0}, y_{0}$, position angle $\phi$, inclination $i$, and rotation speed $V_{c}$ within each annulus. Data near the minor axis are excluded since they have almost no signal due to projection. In practice the kinematic center is not well constrained (see also Begeman 1987, Palunas 1996) and we fixed the central position to be the photometric center 


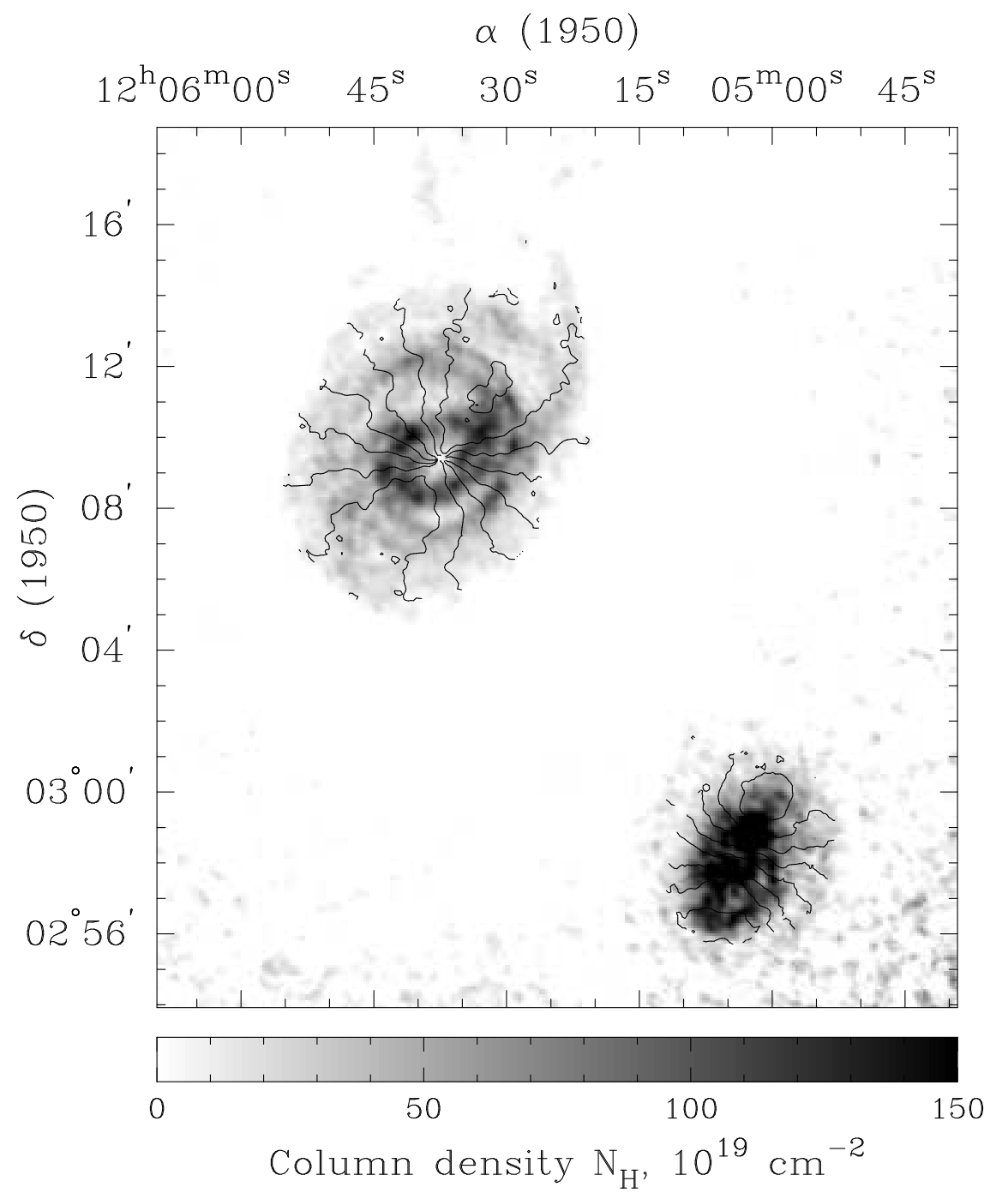

FIG. 7.- VLA map of NGC 4123 and NGC 4116

The $21 \mathrm{~cm}$ surface brightness of NGC 4123 and NGC 4116 is plotted as grayscale, with velocity contours overlaid. North is up and west is to the right. The noise increases in the south and west as these regions are at the edge of the VLA primary beam. The surface brightness grayscale runs from $N_{H}=0$ to $1.5 \times 10^{21} \mathrm{~cm}^{-2}$. The contours are of heliocentric velocity from 1225 to $1425 \mathrm{~km} \mathrm{~s}^{-1}$ at intervals of $25 \mathrm{~km} \mathrm{~s}^{-1}$, and each galaxy has the receding side in the northwest.

of the galaxy.

Rotation curve fits to two-dimensional velocity fields have degeneracies between the parameters, and are influenced by non-circular motions in the disk and warps. We examined the variation of $\phi, i$, and $V_{c}$ with radius. Inside the bar, the parameters are "twisted" by the noncircular motions in the bar. Outside the bar radius, from $\sim 130^{\prime \prime}$ to $250^{\prime \prime}, \phi, i$, and $V_{c}$ change only slowly and we adopted median values of $\phi$ and $i$ from this region, yielding $\phi=-33 \pm 2^{\circ}$ and $i=45 \pm 4^{\circ} . V_{c}(R)$ and $i(R)$ are covariant and show artificial excursions at certain radii, due to spiral arms crossing the annuli (see Palunas 1996). However, by averaging over a radial range we obtain a reliable estimate of the parameters $\phi$ and $i$.

We then extract the rotation curve $V_{c}(R)$ in annuli using the adopted values of $x_{0}, y_{0}, \phi$, and $i$. We chose to use global values of $\phi$ and $i$ rather than doing a tilted-ring extraction, since tilted-ring fits do not work well on galaxies of $i \sim 45^{\circ}$ (e.g. Begeman 1987) and are also thrown off by spiral arms. The rotation curve extraction was spatially oversampled, in part to avoid further smoothing caused by binning into annuli. The residual map in the right panel of Figure 6 shows that the model produces a good representation of the data, justifying the use of global $\phi$ and $i$. Outside the bar region, residuals are generally below 10 $\mathrm{km} \mathrm{s}^{-1}$.

The rotation curve computed from the $\mathrm{H} \mathrm{I}$ data is shown as the points and errorbars in each panel of Figure 8. The approaching and receding sides of the galaxy are plotted separately. The rotation curves for the two sides differ by $10-20 \mathrm{~km} \mathrm{~s}^{-1}$ at large radii; the difference is caused by spiral non-circular streaming motions. The right panel of Figure 6 shows the residual velocity field after subtraction of the axisymmetric rotation curve (using an average of the two sides; the residual map is similar if the approaching and receding sides are subtracted separately). There are strong antisymmetric residuals in the bar region, and a spiral-shaped residual from the outer spiral pattern.

Outside the bar radius of $5.6 \mathrm{kpc}$, it is reasonable to derive a rotation curve assuming that the gas is on circular 

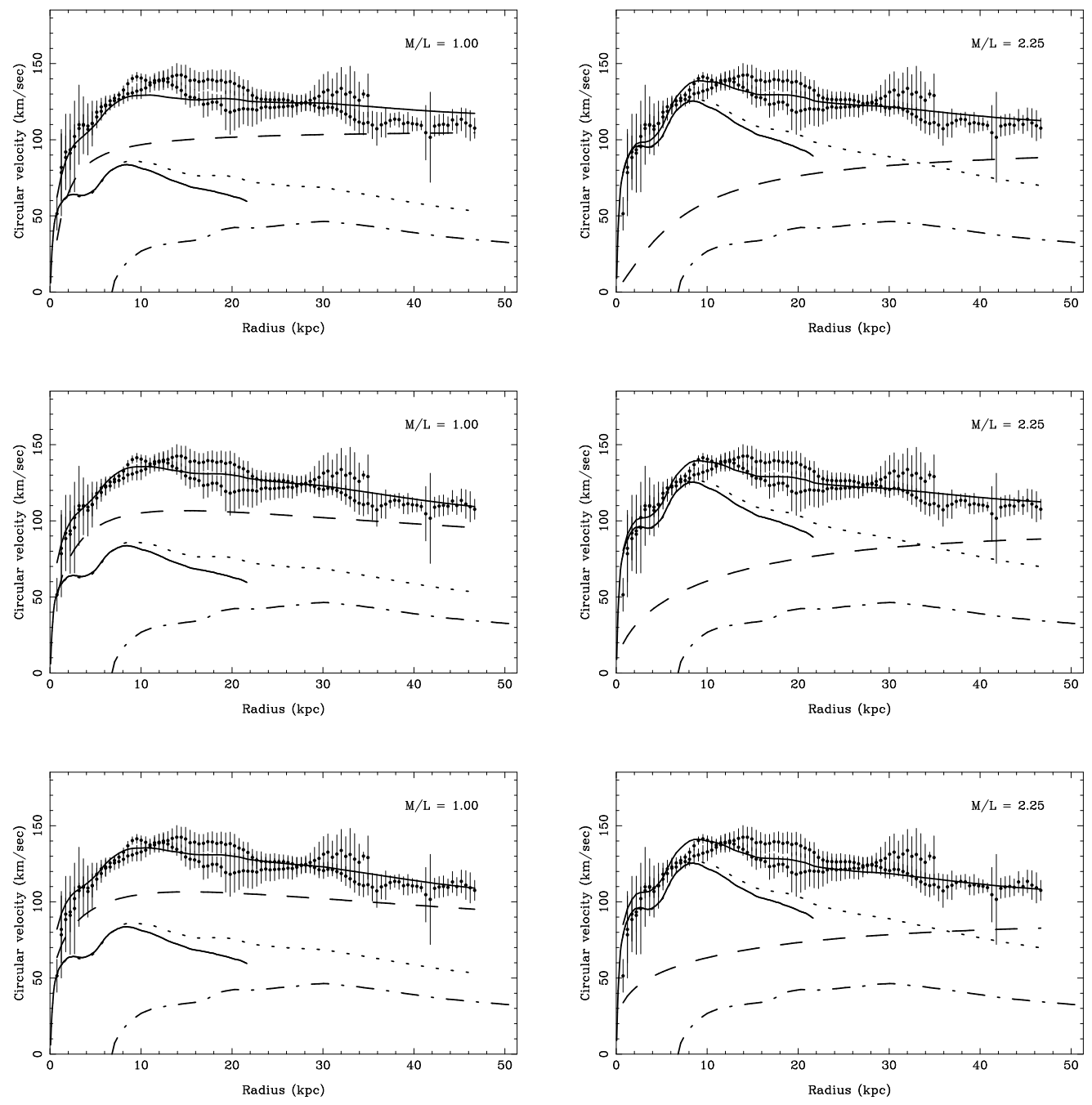

FIG. 8.- Mass models for the rotation curve of NGC 4123

The contributions of the stellar disk, gas disk, and best-fit halo to the rotation curve of NGC 4123 are shown for models with low and high mass disks, and three different dark halo types.

Points and errorbars - observed rotation curve.

Uppermost solid line - best-fit total rotation curve.

Dotted line - contribution of stars+gas to the rotation curve.

Lower solid line - contribution of the stellar disk.

Dashed line - contribution of the best-fit halo.

Dot-dashed line - contribution of the gas disk.

The left column has disk $M / L=1.0$, and the right column has disk $M / L=2.25$. The top row uses isothermal halos, the middle row uses NFW halos, and the bottom row uses Moore-type halos.

orbits. Inside that radius, the derived rotation curve is affected by noncircular motions and the error bars increase because the data are not fully consistent with circular orbits and because the $\mathrm{H}$ I disk has a central hole.

The maximum of the rotation curve is $145 \mathrm{~km} \mathrm{~s}^{-1}$, while the flat part of the rotation curve is at $V_{c}=130 \mathrm{~km} \mathrm{~s}^{-1}$. Comparing the rotation width and total $I$ magnitude to the Tully-Fisher relation of Giovanelli et al. (1997) suggests that the galaxy is overluminous by 0.2 magnitudes, which is consistent with the scatter in that TF relation. The difference could also be caused by a $10 \%$ overestimation of the distance to NGC 4123, which would require a $10 \%$ increase to the $M / L$ values we discuss below.

\subsection{The spiral pattern speed}

The spiral velocity residual is a one-armed pattern that changes sign several times along the arm. An $m=1$ ve- locity residual is expected inside corotation of an $m=2$ spiral pattern (Canzian 1993), but an $m=3$ pattern is expected outside corotation, which suggests that nearly all of the H I disk of NGC 4123 is inside corotation of the spiral. However, the $m=1$ spiral residual is not exactly as in Canzian's model, where the sign of the residual does not change along the arm. Alternatively, if the residual is interpreted as a streaming motion, it is consistent with inward streaming along the arm until the the last sign change, at $200^{\prime \prime}$ along the major axis southeast of the galaxy center. Past this point the positive residual on the approaching, far side of the galaxy indicates outward streaming. The change from inward to outward streaming occurs at the corotation radius, which is then at $21 \mathrm{kpc}$. Again most of the $\mathrm{H}$ I disk is inside corotation.

In Paper II, we show that the corotation radius of the bar is well within the $\mathrm{H} \mathrm{I}$ disk, at 5 to $9 \mathrm{kpc}$. Thus the bar 
and spiral pattern have different corotation radii and different pattern speeds, even though the spiral arms appear to emerge from the ends of the bar. These dynamically determined pattern speeds confirm the suggestion of Sellwood \& Sparke (1988) that bar and spiral pattern speeds in the same galaxy can be significantly different.

\section{MASS MODELS}

\subsection{Fitting the rotation curve}

We fit axisymmetric mass models made up of the stellar disk, gas disk, and a dark halo to the rotation curve. Because they are axisymmetric, these models neglect the bar distortions both in the light and in the velocity field, which are strong only inside the bar radius of $5.6 \mathrm{kpc}$. Our goal is to determine the halo required to fit the outer rotation curve for a range of values for the disk mass-tolight ratio. We also want to determine the maximum disk mass-to-light ratio, the highest disk $M / L$ consistent with the rotation curve data and a non-hollow dark halo.

We calculate the rotation curve of the axisymmetrized stellar disk from the $I$-band image, assuming a uniform $M / L$. Our procedure is described in more detail in $\mathrm{Pa}-$ per II. Briefly, we remove foreground stars and rectify the image to face-on, using the position angle and inclination obtained from the $\mathrm{H}$ I observations. We then average the image in circular annuli about the center to produce an axisymmetrized surface brightness distribution. We assume that the stellar disk has a vertical distribution of the common form $\rho(z) \propto \operatorname{sech}^{2}\left(z / z_{0}\right)$ and a scale height of $z_{0}=400 \mathrm{pc}$, similar to that of the disk of the Milky Way (Mihalas \& Binney 1981). Following Quillen et al. (1994), we calculate the gravitational accelerations as a function of position, summing the contributions of each pixel efficiently using FFT techniques (Hockney 1965). The acceleration as a function of radius determines the stellar contribution $V_{\text {disk }}(R)$ to the rotation curve $V_{c}(R)$.

The rotation curve due to the stellar disk for an assumed $M / L$ is plotted as the lower solid line in the panels of Figure 8. It is plotted out to the end of the optical image only, though its contribution is accounted for outside this radius. Its slightly unusual shape, rising quickly, leveling off, then rising again, is due to the shoulder in the axisymmetrized surface brightness profile (Figure 3) in the bar region.

We calculate the rotation curve due to the gas disk in a similar fashion. We assume that the H I disk is thin and flat and rectify it to face-on, average the column density azimuthally, and scale the mass up by 1.4 to correct for helium. The atomic gas contribution to the rotation curve, shown as the dot-dashed line in Figure 8, is everywhere a rather small fraction of the observed velocity, and inside $7 \mathrm{kpc}$ it makes a very small negative contribution to $V_{c}{ }^{2}$ (i.e. outward centripetal acceleration) due to the central hole in the atomic gas density. The dotted line in Figure 8 is the combined contribution of the stellar and gas disks the contribution of visible, non-dark matter to the rotation curve.

We do not have CO imaging data for NGC 4123, and thus cannot make a detailed calculation of the contribution of its molecular gas to the rotation curve. Single-dish CO observations with the SEST and IRAM 30-m imply a molecular gas mass of $4.0 \times 10^{8} \mathrm{M}_{\odot}$ within a $23^{\prime \prime}$ beam, and
$5.1 \times 10^{8} \mathrm{M}_{\odot}$ within a $45^{\prime \prime}$ beam (Chini, Kruegel \& Steppe 1992). The contribution to the rotation curve from the molecular gas is then $37 \mathrm{~km} \mathrm{~s}^{-1}$ at $1.25 \mathrm{kpc}$ and $30 \mathrm{~km} \mathrm{~s}^{-1}$ at $2.5 \mathrm{kpc}$. The molecular gas may at most fill in the hole in the atomic gas distribution, but its contribution to the overall rotation curve is negligible.

The dark halo needed to fit the observed rotation curve is specified by two parameters: a characteristic density and radius. We performed least-squares fits for these parameters over a range of disk $M / L$ and three different models for the dark halo: a pseudo-isothermal model with a constant density core and $r^{-2}$ envelope:

$$
\rho_{\text {iso }}(r)=\rho_{0} \frac{1}{1+\left(r / r_{c}\right)^{2}}
$$

and two broken power-law models, the "NFW profile" and "Moore profile" found in $N$-body simulations of dark matter halo formation (Navarro, Frenk, \& White 1996; Syer \& White 1998; Moore et al. 1999). The NFW-type model has an inner $r^{-1}$ and outer $r^{-3}$ dependence, and break radius $r_{s}$ :

$$
\rho_{\mathrm{NFW}}(r)=\rho_{s} \frac{4 r_{s}{ }^{3}}{r\left(r+r_{s}\right)^{2}} .
$$

This pseudo-NFW halo model is not precisely an "NFW model," because the NFW family of halos is a one-parameter family: Navarro, Frenk \& White (1996) found a relation between the characteristic density and radius for halos formed in any given cosmology, whereas we allow these parameters to vary independently in order to fit the rotation curve. An NFW-type profile can equivalently be uniquely expressed as a function of concentration index $c$ and virial velocity $V_{200}$ (Navarro et al. 1996). The Mooretype profile is similar to the NFW halo but has an inner cusp slope of $r^{-1.5}$ :

$$
\rho_{\text {Moore }}(r)=\rho_{s} \frac{2}{r / r_{s}{ }^{1.5}\left(1+\left(r / r_{s}\right)^{1.5}\right)} .
$$

Halos with this profile are found in high-resolution $N$-body dark matter simulations (e.g. Moore et al. 1999).

These expressions specify spherical mass distributions, although the rotation curve constrains only the centripetal acceleration in the disk plane; for any degree of halo flattening, a volume density distribution could be devised to yield the same rotation curve. The functional forms used are analytically convenient, but are not intended to favor specific models for halo formation; we have also not attempted to modify the halo profiles for compression by disk collapse, a point we discuss further in Paper II.

The fits are not very sensitive to the functional form adopted: all three types of halo model fit the data well for disk $M / L \leq 2.25$, although for high-mass disks with $M / L \geq 2.0$, the cuspy NFW and Moore halos are forced to very low scale densities and large scale radii in order to contribute to the rotation curve at large radius but avoid exceeding the observations at $R \sim 10 \mathrm{kpc}$.

We constructed models over the range $M / L=1.0$ to 3.0 with the three halo profiles. The models and best-fit halo parameters are listed in Table 4 . For all profile types, the fitted halo density and radius are strongly covariant in a way which keeps the halo contribution to the outer rotation curve roughly constant, although the actual error 
ellipse on the fitted parameters is quite narrow - an example is shown in Paper II. We use the halos fitted in these mass models in the fluid dynamical simulations described in Paper II.

Columns 7 and 8 of Table 4 show the results of fitting NFW-type profiles by fitting for $c$ and $V_{200}$. The best-fit halo profile $\rho(r)$ is the same as that obtained by fitting for $\rho_{s}$ and $r_{s}$, but $c$ and $V_{200}$ are much less covariant than $\rho_{s}$ and $r_{s}$ and are tightly constrained for a given $M / L$. As disk $M / L$ is increased, the halo contribution inside the optical radius decreases greatly and the radius at which the halo rotation curve peaks moves out, requiring a lower concentration index $c$. In order to maintain the necessary halo contribution at large $R$, the virial velocity $V_{200}$ must increase as $c$ decreases; the virial radius $r_{200}$ increases with $V_{200}$. For $M / L \geq 2.0$, the halo rotation curve peaks beyond the end of the $\mathrm{H}$ I data at $47 \mathrm{kpc}$.

\subsection{Discussion}

We plot rotation curve decompositions for $M / L$ of 1.0 and 2.25 in Figure 8. For $M / L \leq 2.25$, all mass models fit the observed rotation curve well and the small differences in the values of reduced $\chi^{2}$, given in Table 4 , are not significant, given that the true shape of the halo radial profile is unknown and could be adjusted slightly to improve the fit.

Models with too high a disk $M / L$ cannot fit well, however, because the fitted rotation curve exceeds the observed curve. The stellar disk alone accounts for the entire rotation curve at $8 \mathrm{kpc}$ when $M / L=2.5$ and the model is an acceptable fit since it only marginally exceeds the data when the isothermal halo is included. For higher $M / L$, the total rotation curve is too high, and the fit is unacceptable. For an isothermal halo, therefore, the maximum disk $M / L$ is about 2.5. NFW and Moore-type halos, which are more centrally concentrated, contribute more to the peak of the rotation curve, and for them the maximum disk $M / L$ is slightly less, about 2.25. For maximum disk models, the NFW and Moore halos have low densities and large scale radii.

\subsection{Radial variation in $M / L$}

It is conventional to assume a constant $M / L$ when fitting rotation curves, but it is not guaranteed, although there is some evidence for constant $M / L$ (van der Kruit 1981). There is a color gradient in NGC 4123, as discussed in Section 3.3, which could be caused by a change in the disk stellar population. To test the effect of a radial variation in $M / L$, we compared the stellar disk contribution to the rotation curve for a constant $M / L$ model and for models in which the $M / L$ declines outside the bar radius. The stellar population is likely to be radially mixed inside the bar radius, and in Paper II we will determine the stellar $M / L$ inside the bar radius, so the important question is whether the rotation curve decomposition is strongly affected by a disk $M / L$ which differs inside and outside the bar radius. Figure 9 shows the disk contribution for three models: a constant $M / L=2.25$ model, and two models with a steep decline in $M / L$ outside the bar radius.

Even these models with a rather exaggerated decline in $M / L$ only have a small effect on the disk contribution to the rotation curve. Reasonable variations in $M / L$ therefore do not substantially increase the amount of dark mat-
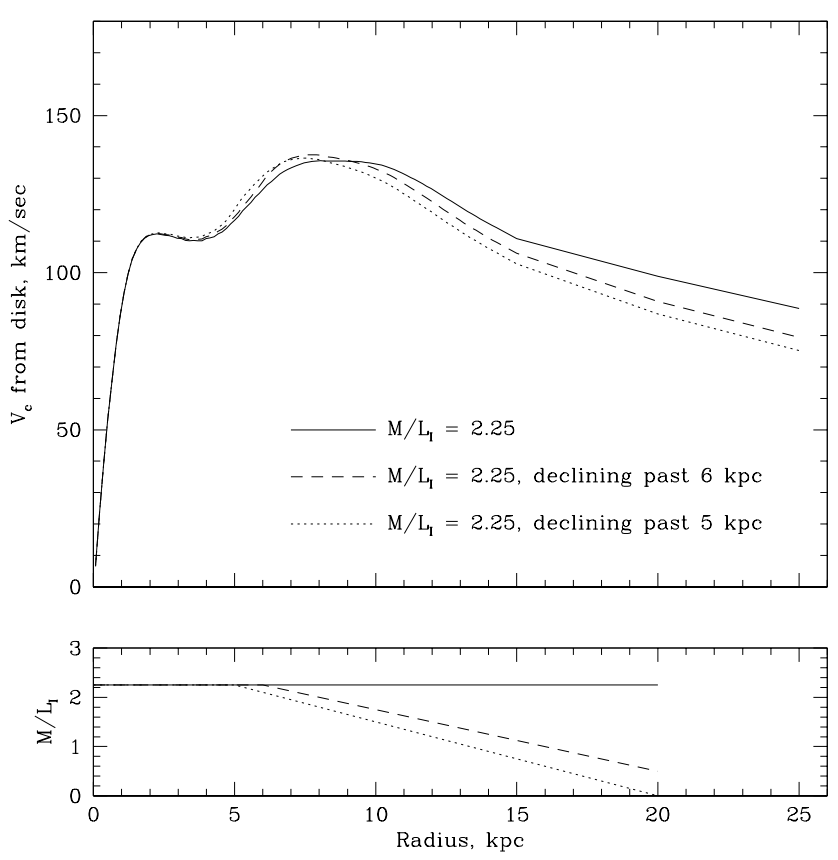

FIG. 9. - Effect of varying $M / L$ on disk rotation curve

The disk contribution to the rotation curve is shown for three models. Solid line: $M / L=2.25$, constant; dashed line: $M / L$ declining outside 6 $\mathrm{kpc}$, to 0.5 at $20 \mathrm{kpc}$; dotted line: $M / L$ declining outside $5 \mathrm{kpc}$, to 0 at $20 \mathrm{kpc}$.

ter required by the rotation curve. An increase in disk $M / L$ with radius, as could happen if the outer disk is bluer because it is old and metal-weak, would of course reduce the required dark matter, but again the effect will be small.

The effect of varying $M / L$ is small because at the bar radius of $5.6 \mathrm{kpc}$, the disk contribution to the rotation curve is already nearing its peak. The decline from the peak of a rotation curve is relatively gradual, whether or not the mass distribution is cut off. A declining $M / L$ outside the bar radius cannot make the rotation curve decline fast enough to change the inferred halo parameters significantly - in fact, a declining $M / L$ makes the disk part of the rotation curve peak up by a tiny amount, because it behaves like a disk truncation.

\section{CONCLUSIONS}

NGC 4123 is a strongly barred galaxy with a rather boxy bar containing two components, both of which are more rectangular than ellipsoidal. The bar has the straight offset dust lanes characteristic of strong bars (Athanassoula 1992b), although they are less obvious in the $I$ image than in bluer bandpasses. The outer disk of the galaxy shows a roughly exponential decline of surface brightness with radius to the limit of the data, at $I \sim 28 ; B, V \sim 29$. The disk extends to at least 10 exponential disk scale lengths; there is no sign that it is truncated, in agreement with 1 mag deeper measurements of NGC 5383 (Barton \& Thompson 1997) but in sharp contrast to the conventional result that disks are truncated at 3-5 scale lengths (cf. van der Kruit \& Searle 1981a,b, 1982; van der Kruit 1989; Barteldrees \& Dettmar 1994). Blue knots in the outer spiral arms indicate that stars are forming well out- 
Weiner et al.

\begin{tabular}{|c|c|c|c|c|c|c|c|c|c|c|c|c|}
\hline \multirow{2}{*}{$\begin{array}{l}\text { Disk } \\
M / L_{I}\end{array}$} & \multicolumn{3}{|c|}{ Isothermal halo } & \multicolumn{3}{|c|}{ NFW-type halo } & \multicolumn{3}{|c|}{ 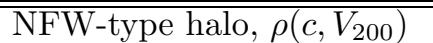 } & \multicolumn{3}{|c|}{ Moore-type halo } \\
\hline & $\rho_{0}$ & $r_{c}$ & $\chi^{2} / N$ & $\rho_{s}$ & $r_{s}$ & $\chi^{2} / N$ & & $c$ & $V_{200}$ & $\rho_{s}$ & $r_{s}$ & $\chi^{2} / N$ \\
\hline 1.00 & 143.0 & 1.24 & 1.76 & 5.47 & 6.82 & 0.71 & 14.2 & \pm 0.09 & $81.2 \pm 0.7$ & 1.60 & 12.1 & 0.76 \\
\hline 1.50 & 97.4 & 1.39 & 0.87 & 1.88 & 10.5 & 0.72 & 9.2 & \pm 0.07 & $81.0 \pm 0.8$ & 0.522 & 19.0 & 0.76 \\
\hline 1.75 & 36.1 & 2.24 & 0.84 & 0.92 & 14.2 & 0.77 & 6.76 & \pm 0.06 & $82.6 \pm 0.9$ & 0.237 & 26.9 & 0.83 \\
\hline 2.00 & 12.7 & 3.76 & 0.87 & 0.39 & 21.4 & 0.85 & 4.35 & \pm 0.04 & $87.5 \pm 1.0$ & 0.0802 & 45.4 & 0.96 \\
\hline 2.25 & 4.68 & 6.33 & 0.93 & 0.13 & 38.2 & 1.02 & 2.09 & \pm 0.03 & $93.0 \pm 1.2$ & 0.0399 & 61.0 & 1.33 \\
\hline 2.50 & 1.91 & 10.4 & 1.11 & 0.043 & 69.1 & 1.40 & 1.30 & \pm 0.01 & $116.3 \pm 1.7$ & 0.0101 & 128.3 & 1.93 \\
\hline 2.75 & 0.88 & 16.9 & 1.63 & 0.011 & 185.7 & 2.10 & 0.645 & \pm 0.005 & $142.8 \pm 2.2$ & 0.0063 & 156.3 & 3.09 \\
\hline 3.00 & 0.46 & 27.1 & 2.75 & 0.0038 & 381.7 & 3.46 & 0.255 & \pm 0.002 & $151.1 \pm 2.9$ & 0.0040 & 179.4 & 4.77 \\
\hline
\end{tabular}

TABLE 4

MASS MODELS: BEST-FIT HALO PARAMETERS

Note. - The best-fit halo parameters for disk mass-to-light ratios from 1.0 to 3.0. Column 1 is the disk $M / L$. Columns 2, 3, and 4 are for isothermal halos; columns 5,6 , and 7 are for NFW-type power-law halos; and columns 10, 11, and 12 are for "Moore-type" power-law halos. The densities are in $10^{-3} \mathrm{M}_{\odot} \mathrm{pc}^{-3}$, and the radii are in kpc. Columns 8 and 9 are the parameters and $1 \sigma$ errors for the best fit NFW-type halos when the fitted parameters are the concentration index $c$ and virial velocity $V_{200}$.

side $R_{25}$.

Fabry-Perot observations of the velocity field show strong non-axisymmetric motions inside the bar radius, and large velocity jumps on the leading edges of the bar, at the location of the dust lanes. These support the interpretation of the dust lanes as the location of shocks in the gas flow. The velocity jumps are offset and several velocity contours make a sharp jog as they passes through the center of the galaxy, suggesting the presence of an inner Lindblad resonance. The strength of the non-axisymmetric motions decreases rapidly beyond the bar radius.

$21 \mathrm{~cm}$ observations of NGC 4123 show that it has an extended gas disk in circular rotation outside the bar radius, with spiral structure visible in the intensity and weakly in the kinematics. The kinematics indicate that corotation of the spiral pattern is at $\sim 4$ times the bar radius; when combined with the result from Paper II that the bar is fast-rotating, this implies that the bar and spiral have very different pattern speeds. There is no evidence for a warp. The rotation curve derived from these observations extends to 4 optical radii $\left(4 R_{25}\right)$ and requires the presence of a dark matter halo in addition to the luminous disk. The maximum height of the rotation curve is $\simeq 145 \mathrm{~km} \mathrm{~s}^{-1}$; the height of the flat part of the rotation curve is $130 \mathrm{~km} \mathrm{~s}^{-1}$.

Mass models that include a stellar disk and dark halo to the $\mathrm{H}$ I rotation curve yield acceptable fits, in a $\chi^{2}$ sense, for $I$-band disk mass-to-light ratios of 2.5 or less. Model halos with either isothermal or power-law density distributions fit the data well. The axisymmetric rotation curve does not contain enough information to constrain the diskhalo decomposition further. Both heavy and light disk models, and both isothermal and power-law dark halos, yield acceptable fits to the rotation curve.

However, there is extra information in the non-circular gas streaming motions seen in the Fabry-Perot data. In Paper II, we use this information to show that the disk of NGC 4123 is close to maximum, with $M / L_{I}=2.25$ the preferred value.

We thank the staff at CTIO, the VLA, and Las Campanas for their excellent support during these observations. BJW thanks Karl Gebhardt and Povilas Palunas for Fabry-Perot reduction software and helpful comments. We thank Jason Prochaska for a reading of the manuscript and the anonymous referee for helpful comments. This research was supported in part by NSF grant AST 96/17088 and NASA LTSA grant NAG 5-6037 to JAS, by NSF grant AST 96-19510 to TBW, and NSF grant AST 96-17177 to JvG. BJW has been supported by a Barbara McClintock postdoctoral fellowship from the Carnegie Institution of Washington. Operation of the RFP is partially supported by CTIO.

\section{REFERENCES}

Athanassoula, E. 1992a, MNRAS, 259, 328

Athanassoula, E. 1992b, MNRAS, 259, 345

Athanassoula, E., Bosma, A., \& Papaioannou, S. 1987, A\&A, 179, 23

Babcock, H.W. 1939, Lick Observatory Bulletin, 19, 41

Bahcall, J.N., \& Casertano, S. 1985, ApJL, 293, L7

Barteldrees, A. \& Dettmar, R.-J. 1994, A\&AS, 103, 475

Barton, I.J., \& Thompson, L.A. 1997, AJ, 114, 655

Beck, R., Ehle, M., Shoutenkov, V., Shukurov, A., \& Sokoloff, D. 1999, Nature, 397, 324

Beers, T.C., Flynn, K., \& Gebhardt, K. 1990, AJ, 100, 32

Begeman, K. 1987, PhD thesis, Groningen

Bosma, A. 1981, AJ 86, 1825

Bosma, A. 1999, in Galaxy Dynamics, ed. Merritt, D.R., Valluri, M., \& Sellwood, J.A. (San Francisco: A.S.P.), 339

Bottema, R. 1993, A\&A, 275, 16

Bottema, R. 1997, A\&A, 328, 517

Briggs, D.S. 1995, PhD thesis, New Mexico Institute of Mining and Technology

Briggs, F.H. 1990, ApJ, 352, 15

Bruzual A., G. \& Charlot, S. 1993, ApJ, 405, 538

Burstein, D., \& Heiles, C. 1982, AJ, 87, 1165

Byun, Y.I., Freeman, K.C. \& Kylafis, N.D. 1994, ApJ, 432, 114

Cardelli, J.A., Clayton, G.C. \& Mathis, J.S. 1989, ApJ, 345, 245

Charlot, S., Worthey, G., \& Bressan, A. 1996, ApJ, 457, 625

Chini, R., Kruegel, E. \& Steppe, H. 1992, A\&A, 255, 87

Courteau, S., \& Rix, H.-W. 1999, ApJ, 513, 561

de Jong, R. 1996a, A\&A, 313, 45 
de Jong, R. 1996b, A\&A, 313, 377

de Vaucouleurs, G. , et al.1991, Third Reference Catalog of Bright Galaxies (RC3), (New York: Springer-Verlag)

Debattista, V.P. \& Sellwood, J.A. 1998, ApJL, 493, L5

Duval, M.F., \& Athanassoula, E. 1983, A\&A, 121, 297

Efstathiou, G., Lake, G. \& Negroponte, J. 1982, MNRAS, 199, 1069

England, M.N. 1989, ApJ, 337, 191

Ferguson, A.M.N., Wyse, R.F.G., Gallagher, J.S. \& Hunter, D.A. 1998, ApJ, 506, L19

Freeman, K.C. 1970, ApJ, 160, 811

Freeman, K.C. 1992, in Physics of Nearby Galaxies: Nature or Nurture?, ed. Thuan, T.X., Balkowski, C., \& Van, J.T.T. (Gif-sur-Yvette: Editions Frontieres), 201

Giovanelli, R., Haynes, M., Salzer, J. Wegner, G., da Costa, L., \& Freudling, W. 1994, AJ, 107, 2036

Giovanelli, R., Haynes, M.P., Herter, T., Vogt, N.P., Wegner, G., Salzer, J.J., Da Costa, L.N., \& Freudling, W. 1997, AJ, 113, 53

Graham, J.A. 1982, PASP, 94, 244

Hockney, R.W. 1965, J. Assoc. Comp. Mach., 12, 95

Huchtmeier, W.K., \& Richter, O.-G. 1989, A General Catalog of H I Observations of Galaxies, (New York: Springer)

Kalnajs, A. 1983, in Internal Kinematics and Dynamics of Disk Galaxies, IAU Symposium 100, ed. Athanassoula, E. (Dordrecht: Reidel), 87

Kennicutt, R. C. , Jr. 1989, ApJ, 344, 685

Landolt, A. U. 1992, AJ, 104, 340

Lindblad, P.O., Hjelm, M., Högbom, J., Jörsater, S., Lindblad, P.A.B., Santos-Lleo, M. 1996a, A\&AS, 120, 403

Lindblad, P.A.B., Lindblad, P.O., \& Athanassoula, E. 1996b, A\&A, 313, 65

Long, K. 1991, PhD thesis, Princeton University

MacAlpine, G.M., \& Williams, G.A. 1981, ApJS, 45, 113

Mihalas, D., \& Binney, J. 1981, Galactic Astronomy, (New York: Freeman)

Moore, B., Quinn, T., Governato, F., Stadel, J. \& Lake, G. 1999, MNRAS, 310,1147

Moore, E.M., \& Gottesman, S.T. 1995, ApJ, 447, 159

Morrison, H.L., Boroson, T.A., \& Harding, P. 1994, AJ, 108, 1191

Navarro, J.F., Frenk, C.S., \& White S.D.M. 1996, ApJ, 462, 563

Navarro, J.F. 1998, preprint astro-ph/9807084

Ondrechen, M.P., \& van der Hulst, J.M. 1989, ApJ, 342, 29

Ondrechen, M.P., van der Hulst, J.M., \& Hummel, E. 1989, ApJ, 342, 39

Opik, E. 1922, ApJ, 55, 406

Palunas, P. 1996, PhD thesis, Rutgers University

Palunas, P., \& Williams, T.B. 2000, ApJ, submitted

Pence, W.D., \& Blackman, C.P. 1984a, MNRAS, 207, 9

Pence, W.D., \& Blackman, C.P. 1984b, MNRAS, 210, 547

Piner, B.G., Stone, J.M., \& Teuben, P.J. 1995, ApJ, 449, 508

Prendergast, K.H. 1966, in Theory of Orbits in the Solar System and in Stellar Systems, IAU Symposium 25, ed. Contopoulos, G., (New York: Academic), 60

Prendergast, K.H. 1983, in Internal Kinematics and Dynamics of Galaxies, IAU Symposium 100, ed. E. Athanassoula, (Dordrecht: Reidel), 215

Press, W.H., Flannery, B.P., Teukolsky, S.A. \& Vetterling, W.T. 1992, Numerical Recipes, (Cambridge: Cambridge U.P.)

Quillen, A.C., Frogel, J.A., \& Gonzalez, R.A. 1 994, ApJ, 437, 162

Quillen, A.C., Frogel, J.A., Kuchinski, L.E. \& Terndrup, D.M. 1995, AJ, 110,156

Regan, M.W., Vogel, S.N., \& Teuben, P.J. 1995, ApJ, 449, 576

Regan, M.W., Vogel, S.N., \& Teuben, P.J. 1997, ApJL, 482, L143

Rhoads, J.E. 1998, AJ, 115, 472

Roberts, M.S. 1969, AJ, 74, 859

Rubin, V.C., Ford, W.K., Jr., \& Thonnard, N. 1978, ApJ, 225, L107

Sandage, A. 1961. The Hubble Atlas of Galaxies, (Washington: Carnegie Inst. of Washington)

Sandage, A., \& Bedke, J. 1988, Atlas of Galaxies: Useful for Measuring the Cosmological Distance Scale, (Washington: NASA, US GPO)

Schommer, R.A., Bothun, G.D., Williams, T.B., \& Mould, J.R. 1993, AJ, $105,97$.

Schwarz, M.P. 1984, MNRAS, 209, 93

Sellwood, J.A. \& Balbus, S.A. 1999, ApJ, 511, 660

Sellwood, J.A. \& Sparke, L.S. 1988, MNRAS, 231, 25P

Syer, D., Mao, S., \& Mo, H.J. 1999, MNRAS, 305, 357

Syer, D. \& White, S.D.M. 1998, MNRAS, 293, 337

Taylor, C.L., Brinks, E., Grashuis, R.M., \& Skillman, E.D. 1995, ApJS, $99,427$.

Tully, R.B., \& Fisher, J.R. 1977, A\&A, 54, 661

van Albada, T.S., Bahcall, J.N., Begeman, K., \& Sancisi, R. 1985, ApJ, 295,305

van Albada, T.S., \& Sancisi, R. 1986, Phil. Trans. Roy. Soc. Lond. A, 320,447

van Albada, T.S., \& Sanders, R.H. 1982, MNRAS, 201, 303.

van der Kruit, P.C. 1981, A\&A, 99, 298

van der Kruit, P.C., \& Searle, L. 1981a, A\&A, 95, 105

van der Kruit, P.C., \& Searle, L. 1981b, A\&A, 95, 116

van der Kruit, P.C., \& Searle, L. 1982, A\&A, 110, 61

van der Kruit, P.C. 1995, in IAU Symposium 164, Stellar Populations,

ed. van der Kruit, P.C. \& Gilmore, G., (Dordrecht: Kluwer), 205

van der Kruit, P.C. 1989, in The World of Galaxies, ed. Corwin, H.G. \& Bottinelli, L. (New York: Springer), 256

Weiner, B.J., \& Sellwood, J.A. 1999, ApJ, 524, 112

Weiner, B.J., Sellwood, J.A., \& Williams, T.B. 2000, ApJ in press (Paper II)

Worthey, G. 1994, ApJS, 95, 107 